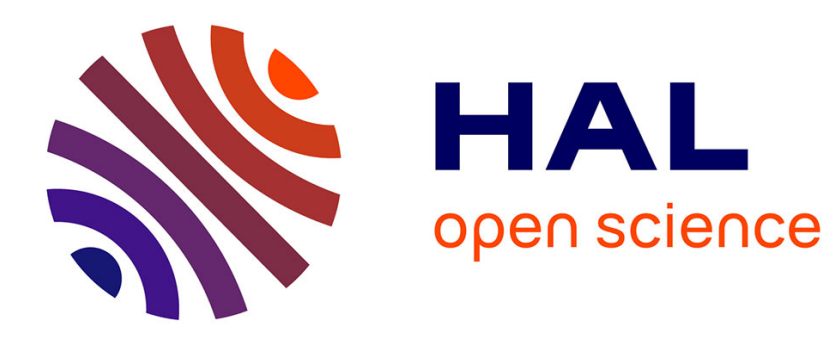

\title{
Decompositions of displacements of thin structures
}

Georges Griso

\author{
- To cite this version: \\ Georges Griso. Decompositions of displacements of thin structures. 2006. hal-00121684
}

\section{HAL Id: hal-00121684 \\ https://hal.science/hal-00121684}

Preprint submitted on 21 Dec 2006

HAL is a multi-disciplinary open access archive for the deposit and dissemination of scientific research documents, whether they are published or not. The documents may come from teaching and research institutions in France or abroad, or from public or private research centers.
L'archive ouverte pluridisciplinaire HAL, est destinée au dépôt et à la diffusion de documents scientifiques de niveau recherche, publiés ou non, émanant des établissements d'enseignement et de recherche français ou étrangers, des laboratoires publics ou privés. 


\title{
Decompositions of displacements of thin structures
}

\author{
G. GRISO
}

\begin{abstract}
In this study we present first the main theorem of the unfolding method in linearized elasticity. Then we prove that every displacement of a thin structure (curved rod or shell) is the sum of an elementary displacement and a warping. Thanks to the previous theorem we obtain sharp estimates of the displacements of this decomposition.
\end{abstract}

Résumé. Dans cette étude on présente d'abord le théorème fondamental de la méthode de l'éclatement en élasticité linéaire. On montre ensuite que tout déplacement d'une structure mince (poutre courbe ou coque) se décompose en la somme d'un déplacement élémentaire et d'un gauchissement. Grâce au théorème fondamental on obtient les estimations des déplacements de cette décomposition.

KEY WORDS: linear elasticity, beams, curved rods, plates, shells, junctions, unfolding method.

\section{Introduction}

Two mathematical methods have been developed to study thin structures. The first one is the formal asymptotic method in which we determine the first term of the asymptotic expansion and then justify the result by an error estimate (see books by J. Sanchez-Hubert and E. Sanchez-Palencia [22] (rods) and Ph. Destuynder [6] (plates)). The second one is the more recent variational asymptotic method in which, for the first time, linearized elasticity of rods, plates or shells has been studied without any geometrical or mechanical a priori hypotheses. This method is presented in works by L. Trabucho and J. M. Viano [24] (rods) and P.G. Ciarlet [4,5] (plates and shells). Our paper is dedicated to a new method in elasticity : the unfolding method in linearized elasticity. The unfolding method offers new tools to study the asymptotic behavior (see $[10,11,14,15]$ ) and the homogenization (see [12]) of structures formed by a large numbers of rods, plates or shells. These tools consist essentially in new decompositions of the displacements of a thin structure and in estimates of the elements of these decompositions. These decompositions make it quite easy to obtain the Korn inequality in a thin domain as well as the asymptotic behavior of the strain tensor of a sequence of displacements. The limits are expressed in terms of formal displacements of the reference structure (see $[10,11,12,13,14,15])$.

Our paper is organised into three parts. Section II is dedicated to Theorem 1.3. This theorem is the main result of the unfolding method in linearized elasticity (see $[10,11,13]$ ). It is a generalization of Lemma 3.1 in [10]. We choose a domain $\mathcal{O}$ in $\mathbb{R}^{n}$ included in a ball of radius less than $R$ and star-shaped with respect to a ball of radius $R_{1}$. Theorem 1.3 gives estimates of the distances for different norms between an element belonging to $W^{1, p}\left(\mathcal{O} ; \mathbb{R}^{n}\right), 1<p<\infty$, and the space of rigid body displacements. The constants appearing in these estimates depend on $n, p$ and on the two parameters $R$ and $R_{1}$ which caracterize the geometry of the open set. To do so, we prove that a displacement $u$ in $W^{1, p}\left(\mathcal{O} ; \mathbb{R}^{n}\right)$ is written $u=v+w$ where $v$ is defined thanks to Newtonian potentials and where $w$ is a harmonic displacement. A rigid displacement $r$ which approximates $u$ is obtained with the mean value of $u$ and the mean value of the skew-symmetric part of $\nabla w$. The estimates of $u-r$ are due to the Poincaré-Wirtinger inequality for functions belonging to $W_{l o c}^{1, p}(\mathcal{O})$ (Proposition 1.2). In fact in Theorem 1.3 we give an upper bound of the distance between the gradient of $u$ and the space of the skew-symmetric matrices with the help of the strain tensor norm of $u$. This theorem can be compare with a result obtained by G. Friesecke, R. D. James and S. Müller which stands out in nonlinear elasticity. In [9] these authors have proved that the distance between the gradient of 
a function $\phi \in H^{1}\left(\mathcal{O} ; \mathbb{R}^{n}\right)$ and $S O(n)$ is estimate by the $L^{2}$ norm of $\operatorname{dist}(\nabla \phi, S O(n))$ and a constant which depends only of the open set $\mathcal{O}$.

The Korn inequality in an open bounded set star-shaped with respect to a ball is an immediate consequence of Theorem 1.3. We also deduce from this theorem Korn's inequality in an open bounded set with lipschitzian boundary.

Several proofs (see $[5,7,20]$ ) of Korn's inequality refer to a lemma of J.-L. Lions $[7,20]$, yet this lemma does not give the dependence of the constant in this inequality with respect to the open set. New proofs of this inequality for displacements in $H^{1}\left(\mathcal{O} ; \mathbb{R}^{n}\right)$ are given in $[2,10,17]$. In these articles we explicitly find the dependence of the constant in Korn's inequality with respect to the open set $\mathcal{O}$. We also find in [19] a proof of Korn's inequality for displacements belonging to $W^{1, p}\left(\Omega ; \mathbb{R}^{n}\right)$ where $\Omega$ is an open bounded set with lipschitzian boundary. But in this paper the authors do not give the dependence of the constant with respect to the open set.

Section III is dedicated to the decompositions of the displacements of a curved rod. The curved rod has a length of $L$ and a thickness of order $\delta$. We define the class of elementary displacements of the curved rod (Definition 2). These displacements are the generalization of the Bernoulli-Navier displacements (see [10]). Then, to any displacement $u$ of the curved rod we associate an elementary displacement $U_{e}$ and a warping $\bar{u}, u=U_{e}+\bar{u}$ (Definition 3). Theorem 2.1 gives sharp estimates with respect to $\delta$ and the strain energy of $u$ for this kind of displacements. In order to study structures made of rods we give, Lemma 2.2, an extension of $u$ at each of its extremities and, Proposition 2.3, a second decomposition of $u$ in the sum of an elementary displacement and a residual one such that this new elementary displacement is equal to a rigid body displacement in a neighborhood of the extremities of the curved rod (see also [18]). Of course all results of this section are true for straight rods.

Section IV is dedicated to the decompositions of the displacements of a shell. The shell has a thickness of $2 \delta$. As in the previous section we define the class of elementary displacements of the shell (Definition 6). These displacements are the generalization of the Kirchhoff-Love displacements (see [11]). Then, to any displacement $u$ of a shell we associate an elementary displacement $U_{e}$ and a warping $\bar{u}, u=U_{e}+\bar{u}$ (Definition 7). Theorem 3.1 gives sharp estimates with respect to $\delta$ and the strain energy of $u$ for this kind of displacements. Our first decomposition of a displacement is the simplest and the most natural. In this decomposition the normal component of the rotation is null. In order to obtain the Korn inequality more easily and to study structures made of several plates or shells we introduce a new decomposition of displacement $u$ into the sum of an elementary displacement and a residual one. In this decomposition the normal component of the rotation vector will not be null any longer (Definition 8) and the estimates of this new decomposition (Theorem 3.3) are simpler than those of Theorem 3.1. To do so we must give, Lemma 3.2 , an extension of $u$ in a neighborhood of thickness $2 \delta$ of the lateral boundary of the shell. Of course all results of this section are true for plates.

The last section is dedicated to two geometrical lemmas and to the proof of Lemma 3.2.

As a rule, the Greek indices $\alpha$ and $\beta$ take values in $\{1,2\}$ and the Latin indices $i, j, k, l$ and $q$ take values in $\{1, \ldots, n\}$.

\section{The fundamental theorem of the unfolding method in linerized elasticity}

\section{Poincaré-Wirtinger's inequality in an open bounded set star-shaped with respect to a ball}

In this section $\mathcal{O}$ denotes an open bounded subset of $\mathbb{R}^{n}$ included in a ball of radius $R$ and star-shaped with respect to the ball $B\left(O ; R_{1}\right)$. The following lemma and proposition are the generalizations of the Lemma 2.6 and Proposition 2.5 in [10]. 
For any $1 \leq p \leq \infty$ and any bounded open set $\Omega$ de $\mathbb{R}^{n}$ we denote

$$
W_{\rho}^{1, p}(\Omega)=\left\{\phi \in L^{p}(\Omega) \mid \rho \nabla \phi \in\left[L^{p}(\Omega)\right]^{n}\right\}
$$

where $\rho(x)$ is the distance between $x$ and the boundary of $\Omega$.

Lemma 1.1 . For any $\phi$ belonging to $W_{\rho}^{1, p}(\mathcal{O}), 1 \leq p<\infty$, we have

$$
\|\phi\|_{L^{p}(\mathcal{O})}^{p} \leq 2^{n+3 p} p^{p}\left\{\frac{R}{R_{1}}\right\}^{n+p-1}\left\{\|\phi\|_{L^{p}\left(B\left(O ; R_{1}\right)\right)}^{p}+\|\rho \nabla \phi\|_{\left[L^{p}(\mathcal{O})\right]^{n}}^{p}\right\}
$$

Proof . Let $\theta$ be a mapping belonging to $\mathcal{C}^{\infty}\left(\mathbb{R}^{+} ;[0,1]\right)$ and such that

$$
\theta(t)=0 \text { if } 0 \leq t \leq \frac{R_{1}}{2} \quad \theta(t)=1 \text { if } R_{1} \leq t \quad\left|\theta^{\prime}(t)\right| \leq \frac{4}{R_{1}} \quad \forall t \in \mathbb{R}^{+}
$$

We recall that for any $\psi \in W^{1, p}(0, a), a>0$, such that $\psi(0)=0$, we have the Hardy's inequality

$$
\int_{0}^{a}|\psi(x)|^{p} d x \leq p^{p} \int_{0}^{a}(a-x)^{p}\left|\psi^{\prime}(x)\right|^{p} d x
$$

Let $\phi$ be in $\mathcal{C}^{\infty}(\overline{\mathcal{O}})$. We consider the segment joining the origin $O$ to an arbitrary point $P$ belonging to the boundary of $\mathcal{O}$. We apply the Hardy inequality to the restriction $\theta(r) \phi(x)$ on this segment. We obtain

$$
\int_{0}^{\|P\|_{2}}|\theta(r) \phi(x)|^{p} d r \leq p^{p} \int_{0}^{\|P\|_{2}}\left|r-\|P\|_{2}\right|^{p}\left|\frac{\partial(\theta \phi)}{\partial r}\right|^{p} d r
$$

where $r=\|x\|_{2}$ (euclidian norm of $x$ ). The open set $\mathcal{O}$ is star-shaped with respect to the ball $B\left(O ; R_{1}\right)$. So we have

$$
\left|r-\|P\|_{2}\right|=\|x-P\|_{2} \leq \frac{R}{R_{1}} \rho(x) \quad \text { and } \quad\left|r-\|P\|_{2}\right| \leq R
$$

From the inequalities (1.2) and (1.3) we deduce

$$
\int_{R_{1}}^{\|P\|_{2}}|\phi(x)|^{p} d r \leq \frac{(2 p R)^{p}}{R_{1}^{p}} \int_{\frac{R_{1}}{2}}^{\|P\|_{2}}|\rho(x) \nabla \phi(x)|^{p} d r+\frac{(8 p R)^{p}}{R_{1}^{p}} \int_{\frac{R_{1}}{2}}^{R_{1}}|\phi(x)|^{p} d r
$$

We multiply the above inequality by $\left\{\frac{R_{1}}{2}\right\}^{n-1}$. Then, in the right hand side we replace this quantity by $r^{n-1}$ and in the left hand side we replace it by $\left(\frac{r}{R} \frac{R_{1}}{2}\right)^{n-1}$. Finally we integrate on all rays (that is to say on the unit sphere of $\mathbb{R}^{n}$ ) to obtain

$$
\int_{\mathcal{O} \backslash B\left(O ; R_{1}\right)}|\phi(x)|^{p} d x \leq 2^{n+3 p-1} p^{p}\left\{\frac{R}{R_{1}}\right\}^{n+p-1}\left\{\|\rho \nabla \phi\|_{\left[L^{p}(\mathcal{O})\right]^{n}}^{p}+\|\phi\|_{L^{p}\left(B\left(O ; R_{1}\right)\right)}^{p}\right\}
$$

To the above inequality we add $\|\phi\|_{L^{p}\left(B\left(O ; R_{1}\right)\right)}^{p}$. We have proved (1.1) for any function $\phi$ belonging to $\mathcal{C}^{\infty}(\overline{\mathcal{O}})$. The space $\mathcal{C}^{\infty}(\overline{\mathcal{O}})$ is dense in $W^{1, p}(\mathcal{O})$ then we obtain $(1.1)$ for any $\phi \in W^{1, p}(\mathcal{O})$.

Now we take $\phi$ in $W_{\rho}^{1, p}(\mathcal{O})$. Let $\mathcal{O}_{\varepsilon}$ be the range of $\mathcal{O}$ in the homothety of center $O$ and ratio $\left.1-\varepsilon, \varepsilon \in\right] 0,1[$. The restriction of $\phi$ to $\mathcal{O}_{\varepsilon}$ belongs to $W^{1, p}\left(\mathcal{O}_{\varepsilon}\right)$, hence

$$
\|\phi\|_{L^{p}\left(\mathcal{O}_{\varepsilon}\right)}^{p} \leq 2^{n+3 p} p^{p}\left\{\frac{R}{R_{1}}\right\}^{n+p-1}\left\{\left\|\left.\rho_{\varepsilon} \nabla \phi\right|_{\left[L^{p}\left(\mathcal{O}_{\varepsilon}\right)\right]^{n}} ^{p}+\right\| \phi \|_{L^{p}\left(B\left(O ;(1-\varepsilon) R_{1}\right)\right)}^{p}\right\}
$$


where $\rho_{\varepsilon}(x)$ is the distance from $x$ to the boundary of $\mathcal{O}_{\varepsilon}$. We have $\rho_{\varepsilon}(x) \leq \rho(x)$. Then (1.1) follows for any $\phi \in W_{\rho}^{1, p}(\mathcal{O})$ by passing to limit as $\varepsilon \rightarrow 0$ and thanks to the Beppo-Levi's Theorem.

We denote $\mathcal{M}_{\Omega}(\phi)$ the mean value of $\phi$ in $\Omega$,

$$
\mathcal{M}_{\Omega}(\phi)=\frac{1}{|\Omega|} \int_{\Omega} \phi \quad \phi \in L^{1}(\Omega), \quad|\Omega|=\operatorname{measure}(\Omega)
$$

Proposition 1.2 . (Poincaré-Wirtinger's inequality.) For any function $\phi$ belonging to $W_{\rho}^{1, p}(\mathcal{O}), 1 \leq p<\infty$, we have

$$
\left\|\phi-\mathcal{M}_{\mathcal{O}}(\phi)\right\|_{L^{p}(\mathcal{O})} \leq p 2^{n+4+n / p}\left\{\frac{R}{R_{1}}\right\}^{\frac{n+p-1}{p}}\|\rho \nabla \phi\|_{\left[L^{p}(\mathcal{O})\right]^{n}}
$$

Proof . Let $\phi$ be in $W_{\rho}^{1, p}(\mathcal{O})$. We apply the Poincaré-Wirtinger inequality to the restriction of $\phi$ to the ball $B\left(O ; R_{1} / 2\right)$ (see $[8]$ chapter 7 )

$$
\left\|\phi-\mathcal{M}_{B\left(O ; R_{1} / 2\right)}(\phi)\right\|_{L^{p}\left(B\left(O ; R_{1} / 2\right)\right)}^{p} \leq 2^{(n-1) p} R_{1}^{p} \int_{B\left(O ; R_{1} / 2\right)}|\nabla \phi(x)|^{p} d x
$$

The distance from a point in $B\left(O ; R_{1} / 2\right)$ and the boundary of $\mathcal{O}$ is more than $R_{1} / 2$, hence

$$
\left\|\phi-\mathcal{M}_{B\left(O ; R_{1} / 2\right)}(\phi)\right\|_{L^{p}\left(B\left(O ; R_{1} / 2\right)\right)}^{p} \leq 2^{n p} \int_{B\left(O ; R_{1} / 2\right)}|\rho(x) \nabla \phi(x)|^{p} d x \leq 2^{n p} \|\left.\rho \nabla \phi\right|_{\left[L^{p}(\mathcal{O})\right]^{n}} ^{p}
$$

We apply Lemma 1.1 to function $\phi-\mathcal{M}_{B\left(O ; R_{1} / 2\right)}(\phi)$ and we use the above inequality. We obtain

$$
\left\|\phi-\mathcal{M}_{B\left(O ; R_{1} / 2\right)}(\phi)\right\|_{L^{p}(\mathcal{O})}^{p} \leq 2^{n p+n+3 p} p^{p}\left\{\frac{R}{R_{1}}\right\}^{n+p-1} \|\left.\rho \nabla \phi\right|_{\left[L^{p}(\mathcal{O})\right]^{n}} ^{p}
$$

We integrate $\phi-\mathcal{M}_{B\left(O ; R_{1} / 2\right)}(\phi)$ on the open set $\mathcal{O}$. We deduce an estimate of $\mathcal{M}_{\mathcal{O}}(\phi)-\mathcal{M}_{B\left(O ; R_{1} / 2\right)}(\phi)$. Then, thanks to the above inequality we obtain the Poincaré-Wirtinger inequality for any function belonging to $W_{\rho}^{1, p}(\mathcal{O})$.

\section{Distances between a displacement and the set of the rigid body displacements}

Let $\Omega$ be an open set in $\mathbb{R}^{n}$. We denote $(1<p<\infty)$

$$
\begin{aligned}
|u|_{\mathcal{D}, \Omega, p}=\|\nabla u\|_{\left[L^{p}(\Omega)\right]^{n^{2}}}, & |u|_{\mathcal{E}, \Omega, p}=\left\|(\nabla u)_{S}\right\|_{\left[L^{p}(\Omega)\right]^{n^{2}}}, \quad u \in W^{1, p}\left(\Omega ; \mathbb{R}^{n}\right) . \\
\mathcal{D}_{p}(u, \Omega)=\|\nabla u\|_{\left[L^{p}(\Omega)\right]^{2}}^{p}, & \mathcal{E}_{p}(u, \Omega)=\left\|(\nabla u)_{S}\right\|_{\left[L^{p}(\Omega)\right]^{n^{2}}}^{p},
\end{aligned}
$$

where $(\nabla u)_{S}$ is the symmetric part of the gradient of $u$ or strain tensor. The elements of this matrix are

$$
\gamma_{i j}(u)=\frac{1}{2}\left\{\frac{\partial u_{i}}{\partial x_{j}}+\frac{\partial u_{j}}{\partial x_{i}}\right\}
$$

We recall that a rigid body displacement $r$ is a function from $\mathbb{R}^{n}$ into itself such that there exist $\mathbf{a} \in \mathbb{R}^{n}$ and a linear mapping $\phi$ whose matrix is skew-symmetric,

$$
r(x)=\mathbf{a}+\phi(x) \quad x \in \mathbb{R}^{n} .
$$


Theorem 1.3 . Let $u$ be a displacement belonging to $W^{1, p}\left(\mathcal{O} ; \mathbb{R}^{n}\right), 1<p<\infty$. There exists a rigid body displacement $r$ such that

$$
|u-r|_{\mathcal{D}, \mathcal{O}, p} \leq C\left\{\frac{R}{R_{1}}\right\}^{\frac{n+p-1}{p}}|u|_{\mathcal{E}, \mathcal{O}, p} \quad\|u-r\|_{L^{p}\left(\mathcal{O} ; \mathbb{R}^{n}\right)} \leq C R\left\{\frac{R}{R_{1}}\right\}^{\frac{n+p-1}{p}}|u|_{\mathcal{E}, \mathcal{O}, p}
$$

The constants depend on $n$ and $p$.

Proof . In every estimate of this proof the constants depend only on $n$ and $p$.

We recall (see [8] chapter 9) that the Newtonian potential $F$ of function $f$ belonging to $L^{p}(\mathcal{O}), 1<p<\infty$, belongs to $W^{2, p}(\mathcal{O})$ and verifies

$$
\Delta F=f \quad \text { in } \quad L^{p}(\mathcal{O}), \quad \int_{\mathcal{O}} \sum_{i, j=1}^{n}\left|\frac{\partial^{2} F}{\partial x_{i} \partial x_{j}}\right|^{p} \leq C\|f\|_{L^{p}(\mathcal{O})}^{p}
$$

Let $u$ be a displacement in $W^{1, p}\left(\mathcal{O} ; \mathbb{R}^{n}\right)$. We denote $v_{i j},(i, j) \in\{1, \ldots, n\}^{2}$, the Newtonian potential of $\gamma_{i j}(u)$. We define the displacement $v$ by

$$
v=\left(v_{1}, \ldots, v_{n}\right) \quad \text { where } \quad v_{k}=\sum_{l=1}^{n}\left\{2 \frac{\partial}{\partial x_{l}} v_{k l}-\frac{\partial}{\partial x_{k}} v_{l l}\right\} \quad k \in\{1, \ldots, n\}
$$

This displacement belongs to $W^{1, p}\left(\mathcal{O} ; \mathbb{R}^{n}\right)$ and verifies $|v|_{\mathcal{E}, \mathcal{O}, p} \leq C|v|_{\mathcal{D}, \mathcal{O}, p} \leq C|u|_{\mathcal{E O}, p}$. Now we consider the displacement $w=u-v$. It belongs to $W^{1, p}\left(\mathcal{O} ; \mathbb{R}^{n}\right)$. From the estimate of $|v|_{\mathcal{E}, \mathcal{O}, p}$ we deduce that $|w|_{\mathcal{E}, \mathcal{O}, p} \leq C|u|_{\mathcal{E}, \mathcal{O}, p}$. We have

$$
\Delta v_{k}=\sum_{l=1}^{n}\left\{2 \frac{\partial}{\partial x_{l}} \gamma_{k l}(u)-\frac{\partial}{\partial x_{k}} \gamma_{l l}(u)\right\}=\Delta u_{k} \quad \text { in } \quad \mathcal{D}^{\prime}(\mathcal{O})
$$

We recall the following classical result about the harmonic functions :

Let $\Omega$ be a bounded domain in $\mathbb{R}^{n}$ with lipschitzian boundary. Any harmonic function u belonging to $L^{p}(\Omega)$, $1 \leq p \leq \infty$, belongs to $W_{\rho}^{1, p}(\Omega)$ and verifies the classical inequality

$$
\|\rho \nabla u\|_{\left[L^{p}(\Omega)\right]^{n}} \leq 4 n\|u\|_{L^{p}(\Omega)}
$$

The components of displacement $w$ are harmonic functions in $\mathcal{O}$ and we have

$$
\frac{\partial^{2} w_{k}}{\partial x_{l} \partial x_{q}}=\frac{\partial}{\partial x_{l}} \gamma_{k q}(w)+\frac{\partial}{\partial x_{q}} \gamma_{k l}(w)-\frac{\partial}{\partial x_{k}} \gamma_{l q}(w) \quad \text { and } \quad \Delta \gamma_{i j}(w)=0 \quad \text { in } \quad \mathcal{O} .
$$

We apply the above estimate to the harmonic functions $\gamma_{i j}(w)$. We obtain

$$
\sum_{i, j=1}^{n}\left\|\rho \frac{\partial^{2} w}{\partial x_{i} \partial x_{j}}\right\|_{L^{p}(\mathcal{O}} \leq C \sum_{i, j=1}^{n}\left\|\rho \nabla \gamma_{i j}(w)\right\|_{\left[L^{p}(\mathcal{O})\right]^{n}} \leq C|w|_{\mathcal{E}, \mathcal{O}, p} \leq C|u|_{\mathcal{E}, \mathcal{O}, p}
$$

Let $\mathcal{A}$ be the skew-symmetric part of matrix $\mathcal{M}_{B\left(O ; R_{1}\right)}(\nabla w)$. The matrix $\mathcal{M}_{B\left(O ; R_{1}\right)}(\nabla w)-\mathcal{A}$ is the mean value in the ball $B\left(O ; R_{1}\right)$ of the symmetric part of the matrix $\nabla w$. We get

$$
\left|\mathcal{M}_{B\left(O ; R_{1}\right)}(\nabla w)-\mathcal{A}\right| \leq C R_{1}^{-\frac{n}{p}}|w|_{\mathcal{E}, \mathcal{O}, p} \leq C R_{1}^{-\frac{n}{p}}|u|_{\mathcal{E}, \mathcal{O}, p}
$$


The estimate of $|v|_{\mathcal{D}, \mathcal{O}, p}$, the Poincaré-Wirtinger inequality (1.5) and inequalities (1.7) and (1.8) give us

$$
\left\{\begin{aligned}
\|\nabla u-\mathcal{A}\|_{\left[L^{p}\left(\mathcal{O} ; \mathbb{R}^{n}\right)\right]^{n}} & \leq\|\nabla v\|_{\left[L^{p}\left(\mathcal{O} ; \mathbb{R}^{n}\right)\right]^{n}}+\left\|\nabla w-\mathcal{M}_{B\left(O ; R_{1}\right)}(\nabla w)\right\|_{\left[L^{p}\left(\mathcal{O} ; \mathbb{R}^{n}\right)\right]^{n}} \\
& +\left|\mathcal{M}_{B\left(O ; R_{1}\right)}(\nabla w)-\mathcal{A} \| \mathcal{O}\right|^{\frac{1}{p}} \leq C\left\{\frac{R}{R_{1}}\right\}^{\frac{n-1}{p}+1}|u|_{\mathcal{E}, \mathcal{O}, p}
\end{aligned}\right.
$$

Now, let $r$ be the rigid body displacement defined by $r(x)=\mathcal{M}_{B\left(O ; R_{1}\right)}(u)+\mathcal{A} x$. Thanks to $(1.9)$ and a last use of the Poincaré-Wirtinger inequality applied to the restriction of function $u-r$ to the ball $B\left(O ; R_{1}\right)$ we obtain an upperbound of $\|u-r\|_{L^{p}\left(\mathcal{O} ; \mathbb{R}^{n}\right)}$.

Corollary 1 . (Korn's inequality in a bounded domain star-shaped with respect to a ball.) Let $u$ be a displacement in $W^{1, p}\left(\mathcal{O} ; \mathbb{R}^{n}\right), 1<p<\infty$. we have

$$
|u|_{\mathcal{D}, \mathcal{O}, p} \leq C\left\{\frac{R}{R_{1}}\right\}^{\frac{2 n+2 p-1}{p}}\left\{\frac{1}{R_{1}}\|u\|_{L^{p}\left(\mathcal{O} ; \mathbb{R}^{n}\right)}+|u|_{\mathcal{E}, \mathcal{O}, p}\right\}
$$

The constant depends on $n$ and $p$.

Proof . Let $u$ be in $W^{1, p}\left(\mathcal{O} ; \mathbb{R}^{n}\right)$. Theorem 1.3 gives a rigid body displacement $r$ verifying estimates (1.6). We have

$$
\|r\|_{L^{p}\left(B\left(O ; R_{1}\right) ; \mathbb{R}^{n}\right)} \leq\|r\|_{L^{p}\left(\mathcal{O} ; \mathbb{R}^{n}\right)} \leq\|u\|_{L^{p}\left(\mathcal{O} ; \mathbb{R}^{n}\right)}+\|u-r\|_{L^{p}\left(\mathcal{O} ; \mathbb{R}^{n}\right)} \leq\|u\|_{L^{p}\left(\mathcal{O} ; \mathbb{R}^{n}\right)}+C R\left\{\frac{R}{R_{1}}\right\}^{\frac{n+p-1}{p}}|u|_{\mathcal{E}, \mathcal{O}, p}
$$

Besides we get

$$
|u|_{\mathcal{D}, \mathcal{O}, p} \leq|u-r|_{\mathcal{D}, \mathcal{O}, p}+|r|_{\mathcal{D}, \mathcal{O}, p} \quad \text { and } \quad|r|_{\mathcal{D}, \mathcal{O}, p} \leq \frac{C}{R_{1}}\left\{\frac{R}{R_{1}}\right\}^{\frac{n}{p}}\|r\|_{L^{p}\left(B\left(O ; R_{1}\right) ; \mathbb{R}^{n}\right)}
$$

From the above inequalities and (1.6) we deduce the estimate of $|u|_{\mathcal{D}, \mathcal{O}, p}$.

Corollary 2. (Korn's inequality.) Let $\Omega$ be a bounded domain in $\mathbb{R}^{n}$ with lipschitzian boundary. For any displacement $u$ in $W^{1, p}\left(\Omega ; \mathbb{R}^{n}\right), 1<p<\infty$, we have

$$
|u|_{\mathcal{D}, \Omega, p} \leq C\left\{\|u\|_{L^{p}\left(\Omega ; \mathbb{R}^{n}\right)}+|u|_{\mathcal{E}, \Omega, p}\right\}
$$

The constant depends on $n, p$ and $\Omega$.

Proof . Any bounded domain in $\mathbb{R}^{n}$ with lipschitzian boundary is the union of a finite sequence of open sets star-shaped with respect to a ball. The estimate of $|u|_{\mathcal{D}, \Omega, p}$ is a consequence of the previous corollary of Theorem 1.3.

\section{Decompositions of the displacements of a curved rod}

\section{Notation}

Let $\zeta$ be a curve in the euclidian space $\mathbb{R}^{3}$ parametrized by its arc length. The current point of the curve is denoted $M\left(s_{3}\right)$ where $s_{3}$ is the arc length.

We suppose that the mapping $s_{3} \longrightarrow M\left(s_{3}\right)$ belongs to $\mathcal{C}^{2}\left(0, L ; \mathbb{R}^{3}\right)$ and that it is injective. We have

$$
\frac{d M}{d s_{3}}=\mathbf{T}, \quad\|\mathbf{T}\|_{2}=1
$$

where $\|\cdot\|_{2}$ is the euclidian norm in $\mathbb{R}^{3}$. 
Let $\mathbf{N}_{1}$ be a function belonging to $\mathcal{C}^{1}\left(0, L ; \mathbb{R}^{3}\right)$ and such that

$$
\forall s_{3} \in[0, L], \quad\left\|\mathbf{N}_{1}\left(s_{3}\right)\right\|_{2}=1 \quad \text { and } \quad \mathbf{T}\left(s_{3}\right) \cdot \mathbf{N}_{1}\left(s_{3}\right)=0
$$

We put

$$
\mathbf{N}_{2}=\mathbf{T} \wedge \mathbf{N}_{1}
$$

In the sequel, $\omega$ denotes a bounded domain in $\mathbb{R}^{2}$ with lipschitzian boundary (while obviously, $\bar{\omega}$ denotes the closure of $\omega)$. We choose the origin $\mathrm{O}$ of coordinates at the center of gravity of $\omega$ and we choose as coordinates axes $\left(O ; \mathbf{e}_{1}\right)$ and $\left(O ; \mathbf{e}_{2}\right)$ the principal axes of inertia of $\omega$. The cross-section $\omega_{\delta}$ of the rod is obtained by transforming $\omega$ with a dilatation of ratio $\delta>0$.

Introduce now the mapping $\Phi: \mathbb{R}^{2} \times[0, L] \longrightarrow \mathbb{R}^{3}$ defined by

$$
\Phi:\left(s_{1}, s_{2}, s_{3}\right) \longmapsto M\left(s_{3}\right)+s_{1} \mathbf{N}_{1}\left(s_{3}\right)+s_{2} \mathbf{N}_{2}\left(s_{3}\right)
$$

There exists $\delta_{0}$ in $\left.] 0, L / 3\right]$ depending only on $\zeta$, such that the restriction of $\Phi$ to the compact set $\bar{\omega}_{\delta_{0}} \times[0, L]$ is a $\mathcal{C}^{1}$ - diffeomorphism of that set onto its range. We can also choose $\delta_{0}$ such that

$$
\forall s \in \Omega_{\delta_{0}}, \quad \frac{1}{2} \leq\|\| \nabla \Phi(s) \|_{2} \leq \frac{3}{2}
$$

where $\|\mid A\|_{2}$ is the spectral norm of the matrix $A$.

Definition 1 . The curved $\operatorname{rod} \mathcal{P}_{\delta}$ is defined as follows :

$$
\left.\left.\left.\mathcal{P}_{\delta}=\Phi\left(\Omega_{\delta}\right), \quad \text { for } \quad \delta \in\right] 0, \delta_{0}\right], \quad \Omega_{\delta}=\omega_{\delta} \times\right] 0, L[
$$

The cross-section of the curved rod is isometric to $\omega_{\delta}$. The point $M\left(s_{3}\right)$ is the center of gravity and the axes of direction $\mathbf{N}_{1}\left(s_{3}\right)$ and $\mathbf{N}_{2}\left(s_{3}\right)$ are the principal axes of inertia of the cross-section of the curved rod.

Notation (i). Reference domains and running points. We denote $x$ the running point of $\mathcal{P}_{\delta}$. The running point of the cylinder $\Omega_{\delta}$ is $s$.

(ii). Displacements. For any displacement $u \in L^{1}\left(\mathcal{P}_{\delta} ; \mathbb{R}^{3}\right)$, we write $u$ instead of $u \circ \Phi$.

\section{First decomposition of displacements of a curved rod.}

Definition 2 . We call elementary displacement of a rod, any element $v$ of the space $L^{1}\left(\Omega_{\delta} ; \mathbb{R}^{3}\right)$ that is written in the form

$$
v(s)=\mathcal{V}\left(s_{3}\right)+\mathcal{A}\left(s_{3}\right) \wedge\left(s_{1} \mathbf{N}_{1}\left(s_{3}\right)+s_{2} \mathbf{N}_{2}\left(s_{3}\right)\right), \quad \text { for } a . \text { e. } s \in \Omega_{\delta}
$$

where $\mathcal{V}$ and $\mathcal{A}$ are functions in $L^{1}\left(0, L ; \mathbb{R}^{3}\right)$.

The first component $\mathcal{V}$ of $v$ is the displacement of the center line. The second component $\mathcal{A}$, gives us an information about the relative displacement of the cross-section $\left\{M\left(s_{3}\right)\right\} \times \omega_{\delta}$ of the rod, that is to say, rotations whose axis are directed along the vector $\mathcal{A}\left(s_{3}\right)$.

To any displacement $u$ of the rod we associate an elementary displacement defined as follows :

Definition 3 . The elementary displacement $U_{e}$, associated to $u \in L^{1}\left(\mathcal{P}_{\delta} ; \mathbb{R}^{3}\right)$, is given by

$$
U_{e}\left(., s_{1}, s_{2}\right)=\mathcal{U}+\mathcal{R} \wedge\left(s_{1} \mathbf{N}_{1}+s_{2} \mathbf{N}_{2}\right), \quad\left(s_{1}, s_{2}\right) \in \omega_{\delta}
$$


where

$$
\left\{\begin{array}{l}
\mathcal{U}=\frac{1}{\delta^{2}|\omega|} \int_{\omega_{\delta}} u\left(s_{1}, s_{2}, .\right) d s_{1} d s_{2}, \\
\mathcal{R} \cdot \mathbf{T}=\frac{1}{\left(I_{1}+I_{2}\right) \delta^{4}} \int_{\omega_{\delta}}\left[\left(s_{1} \mathbf{N}_{1}+s_{2} \mathbf{N}_{2}\right) \wedge u\left(s_{1}, s_{2}, .\right)\right] \cdot \mathbf{T} d s_{1} d s_{2}, \\
\mathcal{R} \cdot \mathbf{N}_{\alpha}=\frac{1}{I_{\alpha} \delta^{4}} \int_{\omega_{\delta}}\left[\left(s_{1} \mathbf{N}_{1}+s_{2} \mathbf{N}_{2}\right) \wedge u\left(s_{1}, s_{2}, .\right)\right] \cdot \mathbf{N}_{\alpha} d s_{1} d s_{2}, \quad I_{\alpha}=\int_{\omega} s_{\alpha}^{2} d s_{1} d s_{2} .
\end{array}\right.
$$

We write

$$
u=U_{e}+\bar{u}
$$

This displacement $\bar{u}$ is the warping.

The next theorem plays a fundamental role in the study of curved rods with the unfolding method. It provides sharp estimates of the components of the elementary displacement $U_{e}$ and of the warping $\bar{u}$ in terms of $\delta$ and of the strain energy of $u$.

Theorem 2.1 . Let $u \in W^{1, p}\left(\mathcal{P}_{\delta} ; \mathbb{R}^{3}\right), 1<p<\infty$, and $u=U_{e}+\bar{u}$ the decomposition of $u$ given by (2.1)-(2.3). There exists $\left.\left.\delta_{1} \in\right] 0, \delta_{0}\right]$ which depends only on $\omega$ and on the middle line of the rod such that for any $\delta \leq \delta_{1}$ the following estimates hold:

$$
\left\{\begin{array}{l}
|\bar{u}|_{\mathcal{D}, \mathcal{P}_{\delta}, p} \leq C|u|_{\mathcal{E}, \mathcal{P}_{\delta}, p}, \quad\|\bar{u}\|_{L^{p}\left(\omega_{\delta} ; \mathbb{R}^{3}\right)} \leq C \delta|u|_{\mathcal{E}, \mathcal{P}_{\delta}, p}, \\
\delta\left\|\frac{d \mathcal{R}}{d s_{3}}\right\|_{L^{p}\left(0, L ; \mathbb{R}^{3}\right)}+\left\|\frac{d \mathcal{U}}{d s_{3}}-\mathcal{R} \wedge \mathbf{T}\right\|_{L^{p}\left(0, L ; \mathbb{R}^{3}\right)} \leq \frac{C}{\delta^{2 / p}}|u|_{\mathcal{E}, \mathcal{P}_{\delta}, p} .
\end{array}\right.
$$

The constants are independent of $\delta$.

Proof . Let $N$ be an integer belonging to $\left[\frac{2 L}{3 \delta}, \frac{L}{\delta}\right]$, and set $\alpha_{k}=k \frac{L}{N}, \quad k \in\{0, \ldots, N\}$.

We have $\delta \leq \frac{L}{N} \leq \frac{3}{2} \delta$. The reference cross-section has a diameter less than $2 R$. We choose $R$ such that $1 \leq R$. Then the domain $\left.\Omega_{\delta, k}=\right] \alpha_{k}, \alpha_{k+1}\left[\times \omega_{\delta}\right.$ has a diameter less than $3 R \delta$. The mapping $s_{3} \longmapsto\left(\mathbf{T}, \mathbf{N}_{1}, \mathbf{N}_{2}\right)$ belongs to $\mathcal{C}^{1}\left(\left[0, L ;\left(\mathbb{R}^{3}\right)^{3}\right)\right.$. Hence there exists $C_{0}>0$ which does not depend on $\delta$ such that

$$
\forall(s, \tilde{s}) \in \Omega_{\delta, k} \times \Omega_{\delta, k}, \quad \quad\|\nabla \Phi(s)-\nabla \Phi(\tilde{s})\|_{2} \leq C_{0} \delta
$$

In the sequel we will work with portions of the $\operatorname{rod} \mathcal{P}_{\delta}$ denoted $\mathcal{P}_{\delta, k}$

$$
\mathcal{P}_{\delta, k}=\Phi\left(\omega_{\delta} \times\right] \alpha_{k}, \alpha_{k+1}[) \quad k \in\{0, \ldots, N-1\}
$$

- First Case . We suppose that the reference cross-section is star-shaped with respect to a disc of radius $R_{1}, 0<R_{1} \leq 1 / 2$. The domain $\Omega_{\delta, k}$ is star-shaped with respect to a ball of radius $R_{1} \delta$. We take $\delta$ such that

$$
\delta \leq \inf \left\{\frac{1}{2.3^{2} C_{0}}\left(\frac{R_{1}}{4(3 R)}\right)^{3}, \delta_{0}\right\}
$$

So we have

$$
C_{0} \delta \leq\left(\frac{R_{1}}{4(3 R)}\right)^{3} \inf _{s \in \Omega_{\delta}}\left\{\|\nabla \Phi(s)\|_{2}^{3}\|\|(\nabla \Phi(s))^{-1} \|_{2}^{2}\right\}
$$

Thanks to the corollary of Lemmas 4.1 and 4.2 (see Section $\mathrm{V}$ ) the domain $\mathcal{P}_{\delta, k}$ has a diameter less than $9 R \delta$ and it is star-shaped with respect to a ball of radius $R_{1} \delta / 8$. 
From Theorem 1.3 there exist rigid body displacements $r_{k}$ such that

$$
\mathcal{D}_{p}\left(u-r_{k}, \mathcal{P}_{\delta, k}\right) \leq C \mathcal{E}_{p}\left(u, \mathcal{P}_{\delta, k}\right), \quad\left\|u-r_{k}\right\|_{L^{p}\left(\mathcal{P}_{\delta, k} ; \mathbb{R}^{3}\right)}^{p} \leq C \delta^{p} \mathcal{E}_{p}\left(u, \mathcal{P}_{\delta, k}\right)
$$

where the constants do not depend on $k$ and $\delta$.

- Second Case . The cross-section is a bounded domain in $\mathbb{R}^{2}$ with lipschitzian boundary. There exists a finite sequence of open sets $\omega^{(1)}, \ldots, \omega^{(K)}$ such that

$$
\omega=\bigcup_{1 \leq l \leq K} \omega^{(l)}, \quad \omega_{\delta}=\bigcup_{1 \leq l \leq K} \omega_{\delta}^{(l)}
$$

and such that every $\omega^{(l)}$ is star-shaped with respect to a disc of radius $R_{1}, 0<R_{1} \leq 1 / 2$. Moreover, the open set $\omega$ is connected, then there exists $\left.\left.R_{2} \in\right] 0, R_{1}\right]$ such that if $\omega^{(r)} \cap \omega^{(s)} \neq \emptyset$ then this intersection contains a disc of radius $R_{2}$.

The domain $\left.\Omega_{\delta, k}^{(l)}=\omega_{\delta}^{(l)} \times\right] \alpha_{k}, \alpha_{k+1}$ [ is star-shaped with respect to a ball of radius $R_{1} \delta$. As in the first case, there exist rigid body displacements $r_{k}^{(l)}$ such that

$$
\mathcal{D}_{p}\left(u-r_{k}^{(l)}, \mathcal{P}_{\delta, k}^{(l)}\right) \leq C \mathcal{E}_{p}\left(u, \mathcal{P}_{\delta, k}^{(l)}\right), \quad\left\|u-r_{k}^{(l)}\right\|_{L^{p}\left(\mathcal{P}_{\delta, k}^{(l)} ; \mathbb{R}^{3}\right)}^{p} \leq C \delta^{p} \mathcal{E}_{p}\left(u, \mathcal{P}_{\delta, k}^{(l)}\right), \quad \mathcal{P}_{\delta, k}^{(l)}=\Phi\left(\Omega_{\delta, k}^{(l)}\right)
$$

If $\omega^{(r)} \cap \omega^{(s)} \neq \emptyset$ the portion $\mathcal{P}_{\delta, k}^{(r)} \cap \mathcal{P}_{\delta, k}^{(s)}$ contains a ball of radius $R_{2} \delta / 8$. This allows us to compare the rigid body displacements $r_{k}^{(r)}$ and $r_{k}^{(s)}$. Eventually we define $r_{k}$ as the mean value of $r_{k}^{(l)}$. We obtain

$$
\mathcal{D}_{p}\left(u-r_{k}, \mathcal{P}_{\delta, k}\right) \leq C \mathcal{E}_{p}\left(u, \mathcal{P}_{\delta, k}\right), \quad\left\|u-r_{k}\right\|_{L^{p}\left(\mathcal{P}_{\delta, k} ; \mathbb{R}^{3}\right)}^{p} \leq C \delta^{p} \mathcal{E}_{p}\left(u, \mathcal{P}_{\delta, k}\right),
$$

The constants do not depend on $k$ and $\delta$. They depend only on $\omega$ and on the middle line of the rod.

Now we go on as in Theorem 3.3 in [10]. Recall that the displacements $r_{k}$ are of the form

$$
r_{k}(x)=A_{k}+B_{k} \wedge x, \quad x \in \mathcal{P}_{\delta, k}, \quad A_{k}, B_{k} \in \mathbb{R}^{3}
$$

Taking the mean value over the cross-sections of the curved rod, and using definition (2.2) of $\mathcal{U}$ and $\mathcal{R}$, we deduce the inequalities

$$
\frac{1}{\delta^{p}}\left\|\mathcal{U}-A_{k}-B_{k} \wedge M\right\|_{L^{p}\left(\alpha_{k}, \alpha_{k+1} ; \mathbb{R}^{3}\right)}^{p}+\left\|\mathcal{R}-B_{k}\right\|_{L^{p}\left(\alpha_{k}, \alpha_{k+1} ; \mathbb{R}^{3}\right)}^{p} \leq \frac{C}{\delta^{2}} \mathcal{E}_{p}\left(u, \mathcal{P}_{\delta, k}\right)
$$

Consequently $\left\|u-U_{e}\right\|_{L^{p}\left(\Omega_{\delta} ; \mathbb{R}^{3}\right)} \leq C \delta|u|_{\mathcal{E}, \mathcal{P}_{\delta}, p}$, where $C$ depends only on the center line of the rod.

Note now that both functions $\mathcal{U}$ and $\mathcal{R}$ belong to $W^{1, p}\left(0, L ; \mathbb{R}^{3}\right)$. We compute the derivatives of the displacement $u$ to get

$$
\frac{\partial u}{\partial s_{\alpha}}=\nabla_{x} u \mathbf{N}_{\alpha}, \quad \frac{\partial u}{\partial s_{3}}=\nabla_{x} u\left[\mathbf{T}+s_{1} \frac{d \mathbf{N}_{1}}{d s_{3}}+s_{2} \frac{d \mathbf{N}_{2}}{d s_{3}}\right]
$$

Taking the restrictions of these derivatives to $\left.\Omega_{\delta, k}=\omega_{\delta} \times\right] \alpha_{k}, \alpha_{k+1}[$, from (2.5) we obtain

$$
\left\|\frac{\partial u}{\partial s_{\alpha}}-B_{k} \wedge \mathbf{N}_{\alpha}\right\|_{L^{p}\left(\Omega_{\delta, k} ; \mathbb{R}^{3}\right)}^{p}+\left\|\frac{\partial u}{\partial s_{3}}-B_{k} \wedge\left[\mathbf{T}+s_{1} \frac{d \mathbf{N}_{1}}{d s_{3}}+s_{2} \frac{d \mathbf{N}_{2}}{d s_{3}}\right]\right\|_{L^{p}\left(\Omega_{\delta, k} ; \mathbb{R}^{3}\right)}^{p} \leq C \mathcal{E}_{p}\left(u, \mathcal{P}_{\delta, k}\right)
$$


Then, from (2.6) and by eliminating $B_{k}$ we get

$$
\left\|\frac{\partial u}{\partial s_{\alpha}}-\mathcal{R} \wedge \mathbf{N}_{\alpha}\right\|_{L^{p}\left(\Omega_{\delta} ; \mathbb{R}^{3}\right)}+\left\|\frac{\partial u}{\partial s_{3}}-\mathcal{R} \wedge\left[\mathbf{T}+s_{1} \frac{d \mathbf{N}_{1}}{d s_{3}}+s_{2} \frac{d \mathbf{N}_{2}}{d s_{3}}\right]\right\|_{L^{p}\left(\Omega_{\delta} ; \mathbb{R}^{3}\right)} \leq C|u|_{\mathcal{E}, \mathcal{P}_{\delta}, p} .
$$

By taking the mean value of $\frac{\partial u}{\partial s_{3}}-\mathcal{R} \wedge\left[\mathbf{T}+s_{1} \frac{d \mathbf{N}_{1}}{d s_{3}}+s_{2} \frac{d \mathbf{N}_{2}}{d s_{3}}\right]$ over the cross-section of the rod we obtain

$$
\left\|\frac{d \mathcal{U}}{d s_{3}}-\mathcal{R} \wedge \mathbf{T}\right\|_{L^{p}\left(0, L ; \mathbb{R}^{3}\right)} \leq \frac{C}{\delta^{2 / p}}|u|_{\mathcal{E}, \mathcal{P}_{\delta}, p}
$$

We now estimate the $L^{p}$ norm of $\frac{d \mathcal{R}}{d s_{3}}$. To begin with, let us introduce the function

$$
V\left(s_{3}\right)=\frac{1}{\delta^{4}} \int_{\omega_{\delta}}\left(s_{1} \mathbf{N}_{1}\left(s_{3}\right)+s_{2} \mathbf{N}_{2}\left(s_{3}\right)\right) \wedge u(s) d s_{1} d s_{2} .
$$

We derive $V$ with respect to $s_{3}$

$$
\frac{d V}{d s_{3}}=\frac{1}{\delta^{4}} \int_{\omega_{\delta}}\left[\left(s_{1} \mathbf{N}_{1}+s_{2} \mathbf{N}_{2}\right) \wedge \frac{\partial u}{\partial s_{3}}(s)+\left(s_{1} \frac{d \mathbf{N}_{1}}{d s_{3}}+s_{2} \frac{d \mathbf{N}_{2}}{d s_{3}}\right) \wedge u(s)\right] d s_{1} d s_{2} .
$$

We replace $\frac{\partial u}{\partial s_{3}}$ by $\mathcal{R} \wedge\left[\mathbf{T}+s_{1} \frac{d \mathbf{N}_{1}}{d s_{3}}+s_{2} \frac{d \mathbf{N}_{2}}{d s_{3}}\right]$ and $u$ by $U_{e}$. We obtain (summation on $\alpha$ )

$$
\left\|\frac{d V}{d s_{3}}+I_{\alpha}\left(\mathcal{R} \cdot \mathbf{N}_{\alpha}\right) \frac{d \mathbf{N}_{\alpha}}{d s_{3}}+I_{\alpha}\left(\mathcal{R} \cdot \frac{d \mathbf{N}_{\alpha}}{d s_{3}}\right) \mathbf{N}_{\alpha}\right\|_{L^{p}\left(0, L ; \mathbb{R}^{3}\right)} \leq \frac{C}{\delta^{1+2 / p}}|u|_{\mathcal{E}, \mathcal{P}_{\delta}, p}
$$

The derivatives of the functions $\mathbf{T}, \mathbf{N}_{1}$ and $\mathbf{N}_{2}$ are

$$
\frac{d \mathbf{T}}{d s_{3}}=a_{1} \mathbf{N}_{1}+a_{2} \mathbf{N}_{2} \quad \frac{d \mathbf{N}_{1}}{d s_{3}}=-a_{1} \mathbf{T}+b \mathbf{N}_{2} \quad \frac{d \mathbf{N}_{2}}{d s_{3}}=-a_{2} \mathbf{T}-b \mathbf{N}_{1}
$$

where $a_{\alpha}$ and $b$ are continuous functions defined on $[0, L]$. Hence (no summation on $\alpha$ )

$$
\begin{array}{r}
\left\|\frac{d V}{d s_{3}} \cdot \mathbf{T}-a_{1} I_{1}\left(\mathcal{R} \cdot \mathbf{N}_{1}\right)-a_{2} I_{2}\left(\mathcal{R} \cdot \mathbf{N}_{2}\right)\right\|_{L^{p}(0, L)} \leq \frac{C}{\delta^{1+2 / p}}|u|_{\mathcal{E}, \mathcal{P}_{\delta}, p} \\
\left\|\frac{d V}{d s_{3}} \cdot \mathbf{N}_{\alpha}-a_{\alpha} I_{\alpha}(\mathcal{R} \cdot \mathbf{T})+b\left(I_{1}-I_{2}\right)\left(\mathcal{R} \cdot \mathbf{N}_{3-\alpha}\right)\right\|_{L^{p}(0, L)} \leq \frac{C}{\delta^{1+2 / p}}|u|_{\mathcal{E}, \mathcal{P}_{\delta}, p} .
\end{array}
$$

Using the definition of $\mathcal{R}$ and $V$, one can write (summation on $\alpha)\left(I_{1}+I_{2}\right) \mathcal{R}=V+\frac{I_{\alpha}}{I_{3-\alpha}}\left(V \cdot \mathbf{N}_{\alpha}\right) \mathbf{N}_{\alpha}$ and so (summation on $\alpha$ )

$$
\left(I_{1}+I_{2}\right) \frac{d \mathcal{R}}{d s_{3}}=\frac{d V}{d s_{3}}+\frac{I_{\alpha}}{I_{3-\alpha}}\left[\left(\frac{d V}{d s_{3}} \cdot \mathbf{N}_{\alpha}\right) \mathbf{N}_{\alpha}+\left(V \cdot \frac{d \mathbf{N}_{\alpha}}{d s_{3}}\right) \mathbf{N}_{\alpha}+\left(V \cdot \mathbf{N}_{\alpha}\right) \frac{d \mathbf{N}_{\alpha}}{d s_{3}}\right] .
$$

We deduce that (no summation on $\alpha$ )

$$
\begin{aligned}
\left(I_{1}+I_{2}\right) \frac{d \mathcal{R}}{d s_{3}} \cdot \mathbf{T} & =\frac{d V}{d s_{3}} \cdot \mathbf{T}-\frac{a_{1} I_{1}}{I_{2}}\left(V \cdot \mathbf{N}_{1}\right)-\frac{a_{2} I_{2}}{I_{1}}\left(V \cdot \mathbf{N}_{2}\right) \\
I_{3-\alpha} \frac{d \mathcal{R}}{d s_{3}} \cdot \mathbf{N}_{\alpha} & =\frac{d V}{d s_{3}} \cdot \mathbf{N}_{\alpha}-\frac{a_{\alpha} I_{\alpha}}{I_{1}+I_{2}}(V \cdot \mathbf{T})+\frac{b\left(I_{1}-I_{2}\right)}{I_{\alpha}}\left(V \cdot \mathbf{N}_{3-\alpha}\right)
\end{aligned}
$$


Hence $\delta\left\|\frac{d \mathcal{R}}{d s_{3}}\right\|_{L^{p}\left(0, L ; \mathbb{R}^{3}\right)} \leq \frac{C}{\delta^{2 / p}}|u|_{\mathcal{E}, \mathcal{P}_{\delta}, p}$. Now, observe that

$$
\begin{aligned}
\frac{\partial}{\partial s_{\alpha}}\left(u-U_{e}\right) & =\frac{\partial u}{\partial s_{\alpha}}-\mathcal{R} \wedge \mathbf{N}_{\alpha} \\
\frac{\partial}{\partial s_{3}}\left(u-U_{e}\right) & =\frac{\partial u}{\partial s_{3}}-\frac{d \mathcal{U}}{d s_{3}}-\mathcal{R} \wedge\left[s_{1} \frac{d \mathbf{N}_{1}}{d s_{3}}+s_{2} \frac{d \mathbf{N}_{2}}{d s_{3}}\right]-\frac{d \mathcal{R}}{d s_{3}} \wedge\left(s_{1} \mathbf{N}_{1}+s_{2} \mathbf{N}_{2}\right) \\
& =\frac{\partial u}{\partial s_{3}}-\mathcal{R} \wedge\left[\mathbf{T}+s_{1} \frac{d \mathbf{N}_{1}}{d s_{3}}+s_{2} \frac{d \mathbf{N}_{2}}{d s_{3}}\right]-\left(\frac{d \mathcal{U}}{d s_{3}}-\mathcal{R} \wedge \mathbf{T}\right)-\frac{d \mathcal{R}}{d s_{3}} \wedge\left(s_{1} \mathbf{N}_{1}+s_{2} \mathbf{N}_{2}\right) .
\end{aligned}
$$

From these expressions and taking into account the above inequalities, we finally obtain the estimate of gradient $\bar{u}$.

Remarks . i) We have

$$
\left|U_{e}\right|_{\mathcal{E}, \mathcal{P}_{\delta}, p} \leq\left|U_{e}+\bar{u}\right|_{\mathcal{E}, \mathcal{P}_{\delta}, p}+|\bar{u}|_{\mathcal{E}, \mathcal{P}_{\delta}, p} \leq|u|_{\mathcal{E}, \mathcal{P}_{\delta}, p}+|\bar{u}|_{\mathcal{D}, \mathcal{P}_{\delta}, p} \leq C|u|_{\mathcal{E}, \mathcal{P}_{\delta}, p}
$$

By an easy computation we can see that this estimate of $\left|U_{e}\right|_{\mathcal{E}, \mathcal{P}_{\delta}, p}$ is equivalent to

$$
\delta\left\|\frac{d \mathcal{R}}{d s_{3}}\right\|_{L^{p}\left(0, L ; \mathbb{R}^{3}\right)}+\left\|\frac{d \mathcal{U}}{d s_{3}}-\mathcal{R} \wedge \mathbf{T}\right\|_{L^{p}\left(0, L ; \mathbb{R}^{3}\right)} \leq \frac{C}{\delta^{2 / p}}|u|_{\mathcal{E}, \mathcal{P}_{\delta}, p}
$$

ii) If the rod is clamped at one of these extremities, $\{0\} \times \omega_{\delta}$ for example, we have $\mathcal{R}(0)=\mathcal{U}(0)=0$.

Corollary of Theorem 2.1 . (Korn's inequality). We suppose that the rod is clamped at one of these extremities, then we have

$$
|u|_{\mathcal{D}, \mathcal{P}_{\delta}, p}+\|\left. u\right|_{L^{p}\left(\mathcal{P}_{\delta} ; \mathbb{R}^{3}\right)} \leq \frac{C}{\delta}|u|_{\mathcal{E}, \mathcal{P}_{\delta}, p}
$$

The constant does not depend on $\delta$.

Proof . This is an immediate consequence of (2.4).

\section{Second decomposition of displacements of a curved rod.}

In this paragraph we suppose that the reference cross-section $\omega$ is of diameter $2 R$ and that it is starshaped with respect to a disc centered in the origin and of radius $R_{1}, R_{1} \leq 1 / 2$ and $R \geq 1$.

Now we suppose that the mapping $\Phi$ introduced in the previous subsection is defined on $[-l, L+l] \times \bar{\omega}_{\delta_{0}}$, $l>0$, and it is a $\mathcal{C}^{1}-$ diffeomorphism of that set onto its range and verifies

$$
\forall s \in[-l, L+l] \times \bar{\omega}_{\delta_{0}}, \quad \frac{1}{2} \leq\|\| \nabla \Phi(s) \|_{2} \leq \frac{3}{2}
$$

Lemma 2.2 . Let $\rho$ be a strictly positive real and $u$ a displacement belonging to $W^{1, p}\left(\mathcal{P}_{\delta} ; \mathbb{R}^{3}\right), 1<p<\infty$. There exists an extension $P(u)$ of $u$ to the curved rod

$$
\mathcal{P}_{\delta}^{\prime}=\Phi(]-\rho \delta, L+\rho \delta\left[\times \omega_{\delta}\right) \quad(\delta \leq l / \rho)
$$

such that

$$
P(u) \in W^{1, p}\left(\mathcal{P}_{\delta}^{\prime} ; \mathbb{R}^{3}\right) \quad P(u)_{\left.\right|_{\mathcal{P}_{\delta}}}=u \quad|P(u)|_{\mathcal{E}, \mathcal{P}_{\delta}^{\prime}, p} \leq C|u|_{\mathcal{E}, \mathcal{P}_{\delta}, p}
$$

The constant does not depend on $\delta$. 
Proof . The domain $]-\rho \delta,(2+\rho) \delta\left[\times \omega_{\delta}\right.$ has a diameter less than $2(R+1+\rho) \delta$ and it is star-shaped with respect to a ball of radius $R_{1} \delta$. We put

$$
\mathcal{P}_{0, \rho \delta}=\Phi(] 0,(2+\rho) \delta\left[\times \omega_{\delta}\right) \quad \mathcal{P}_{0, \rho \delta}^{\prime}=\Phi(]-\rho \delta, 0\left[\times \omega_{\delta}\right) .
$$

From Theorem 1.3, there exists a rigid body displacement $r$ such that

$$
\mathcal{D}_{p}\left(u-r, \mathcal{P}_{0, \rho \delta}\right) \leq C \mathcal{E}_{p}\left(u, \mathcal{P}_{0, \rho \delta}\right), \quad\|u-r\|_{L^{p}\left(\mathcal{P}_{0, \rho \delta} ; \mathbb{R}^{3}\right)}^{p} \leq C \delta^{p} \mathcal{E}_{p}\left(u, \mathcal{P}_{0, \rho \delta}\right),
$$

where the constants do not depend on $\delta$. Now we consider the restriction of $(u-r) \circ \Phi$ to the domain ] $0,(2+\rho) \delta\left[\times \omega_{\delta}\right.$. By a reflexion with respect to the plane $s_{3}=0$ we obtain an extension of $(u-r) \circ \Phi$ on the open set $]-\rho \delta, 0\left[\times \omega_{\delta}\right.$. Hence an extension $v$ of $u-r$ on the portion $\mathcal{P}_{0, \rho \delta}^{\prime}$ of the curved rod. We define $P(u)$ by $P(u)=v+r$. We do the same with the other extremity of the curved rod.

From now on any displacement belonging to $W^{1, p}\left(\mathcal{P}_{\delta} ; \mathbb{R}^{3}\right)$ is extended to a displacement belonging to $W^{1, p}\left(\mathcal{P}_{\delta}^{\prime} ; \mathbb{R}^{3}\right)$. We denote again $u$ this extension.

We denote $B_{1}$ the ball centered at $O$ and of radius $R_{1} / 16$. We put

$$
\left|B_{1}\right|=\int_{B_{1}} d x \quad M_{1}=\int_{B_{1}}\|x\|_{2}^{2} d x
$$

Notice that for all $s_{3} \in[0, L]$ the ball centered in $M\left(s_{3}\right)$ and of radius $R_{1} / 16$ is included in $\mathcal{P}_{\delta}^{\prime}$.

To any displacement $u$ of the curved rod we associate a new elementary displacement defined as follows :

Definition 4 . The elementary displacement $U^{\prime}$, associated to $u \in L^{1}\left(\mathcal{P}_{\delta}^{\prime} ; \mathbb{R}^{3}\right)$, is given by

$$
\left.U_{e}^{\prime}(s)=\mathcal{U}^{\prime}\left(s_{3}\right)+\mathcal{R}^{\prime}\left(s_{3}\right) \wedge\left(s_{2} \mathbf{N}_{1}\left(s_{3}\right)+s_{1} \mathbf{N}_{2}\left(s_{3}\right)\right), \quad \text { for } a . \text { e. } s \in\right]-\rho \delta, L+\rho \delta\left[\times \omega_{\delta},\right.
$$

where

$$
\left\{\begin{array}{l}
\mathcal{U}^{\prime}\left(s_{3}\right)=\frac{1}{\left|B_{1}\right|} \int_{B_{1}} u\left(M\left(s_{3}\right)+\delta x\right) d x, \\
\mathcal{R}^{\prime}\left(s_{3}\right)=\frac{3}{2 M_{1} \delta} \int_{B_{1}} x \wedge u\left(M\left(s_{3}\right)+\delta x\right) d x, \\
\mathcal{U}^{\prime}\left(s_{3}\right)=\mathcal{U}^{\prime}(0)+\mathcal{R}^{\prime}(0) \wedge\left(M\left(s_{3}\right)-M(0)\right) \\
\left.\mathcal{U}^{\prime}\left(s_{3}\right)=s_{3} \in[0, L],-\rho \delta, 0\right], \\
\mathcal{U}^{\prime}(L)+\mathcal{R}^{\prime}(L) \wedge\left(M\left(s_{3}\right)-M(L)\right) \quad s_{3} \in[L, L+\rho \delta[, \\
\left.\left.\mathcal{R}^{\prime}\left(s_{3}\right)=\mathcal{R}^{\prime}(0) \quad s_{3} \in\right]-\rho \delta, 0\right], \quad \mathcal{R}^{\prime}\left(s_{3}\right)=\mathcal{R}^{\prime}(L) \quad s_{3} \in[L, L+\rho \delta[.
\end{array}\right.
$$

We write

$$
u=U_{e}^{\prime}+\bar{u}^{\prime}
$$

The elementary displacement $U_{e}^{\prime}$ is a rigid body displacement in the additional parts of the curved rod.

Proposition 2.3 . Let $u \in W^{1, p}\left(\mathcal{P}_{\delta} ; \mathbb{R}^{3}\right), 1<p<\infty$, and $u=U_{e}^{\prime}+\bar{u}^{\prime}$ the decomposition of the extension of u given by (2.7)-(2.9). There exists $\left.\left.\delta_{1}^{\prime} \in\right] 0, \delta_{0}\right]$ which depends only on $\omega, \rho$ and on the middle line of the rod such that for any $\delta \leq \delta_{1}^{\prime}$ the following estimates hold:

$$
\left\{\begin{array}{l}
\left|\bar{u}^{\prime}\right|_{\mathcal{D}, \mathcal{P}_{\delta}^{\prime}, p} \leq C|u|_{\mathcal{E}, \mathcal{P}_{\delta}, p}, \quad\left\|\bar{u}^{\prime}\right\|_{L^{p}\left(\mathcal{P}_{\delta}^{\prime} ; \mathbb{R}^{3}\right)} \leq C \delta|u|_{\mathcal{E}, \mathcal{P}_{\delta}, p}, \\
\delta\left\|\frac{d \mathcal{R}^{\prime}}{d s_{3}}\right\|_{L^{p}\left(0, L ; \mathbb{R}^{3}\right)}+\left\|\frac{d \mathcal{U}^{\prime}}{d s_{3}}-\mathcal{R}^{\prime} \wedge \mathbf{T}\right\|_{L^{p}\left(0, L ; \mathbb{R}^{3}\right)} \leq \frac{C}{\delta^{2 / p}}|u|_{\mathcal{E}, \mathcal{P}_{\delta}, p} .
\end{array}\right.
$$


The constants are independent of $\delta$.

Proof . Let $N$ and $\left(\alpha_{k}\right)_{0 \leq k \leq N}$ be the integer and the sequence introduced in Theorem 2.1. We put

$$
\alpha_{-1}=-\rho \delta \quad \alpha_{N+1}=L+\rho \delta
$$

We denote $\mathcal{P}_{\delta, k}^{\prime}$ the rod portions

$\mathcal{P}_{\delta, 0}^{\prime}=\Phi\left(\omega_{\delta} \times\right]-\rho \delta, 2 \delta[) \quad \mathcal{P}_{\delta, k}^{\prime}=\Phi\left(\omega_{\delta} \times\right](k-1) \delta,(k+2) \delta[) \quad 1 \leq k \leq N-1, \quad \mathcal{P}_{\delta, N}^{\prime}=\Phi\left(\omega_{\delta} \times\right] L-\delta, L+\rho \delta[)$

From Theorem 1.3 there exist rigid body displacements $r_{k}, 0 \leq k \leq N$, such that

$$
\begin{aligned}
\mathcal{D}_{p}\left(u-r_{k}, \mathcal{P}_{\delta, k}^{\prime}\right) & \leq C \mathcal{E}_{p}\left(u, \mathcal{P}_{\delta, k}^{\prime}\right), \\
\left\|u-r_{k}\right\|_{L^{p}\left(\mathcal{P}_{\delta, k}^{\prime} ; \mathbb{R}^{3}\right)} & \leq C \delta^{p} \mathcal{E}_{p}\left(u, \mathcal{P}_{\delta, k}^{\prime}\right), \quad r_{k}(x)=A_{k}+B_{k} \wedge x, \quad x \in \mathcal{P}_{\delta, k}^{\prime}, \quad A_{k}, B_{k} \in \mathbb{R}^{3} .
\end{aligned}
$$

Now we go on as in proof of Theorem 2.1. Using definition (2.8) of $\mathcal{U}^{\prime}$ and $\mathcal{R}^{\prime}$ we obtain $\left(s_{3} \in[0, L]\right)$

$$
\begin{aligned}
\left|\mathcal{U}^{\prime}\left(s_{3}\right)-A_{k}-B_{k} \wedge M\left(s_{3}\right)\right|^{p} & \leq \frac{1}{\left|B_{1}\right|} \int_{B_{1}}\left|\left(u-r_{k}\right)\left(M\left(s_{3}\right)+\delta x\right)\right|^{p} d x \leq \frac{1}{\delta^{3}\left|B_{1}\right|} \int_{\mathcal{P}_{\delta, k}^{\prime}}\left|\left(u-r_{k}\right)(x)\right|^{p} d x \\
\left|\mathcal{R}^{\prime}\left(s_{3}\right)-B_{k}\right|^{p} & \leq \frac{C}{\delta^{p}} \int_{B_{1}}\left|\left(u-r_{k l}\right)\left(M\left(s_{3}\right)+\delta x\right)\right|^{p} d x \leq \frac{C}{\delta^{p+3}} \int_{\mathcal{P}_{\delta, k}^{\prime}}\left|\left(u-r_{k}\right)(x)\right|^{p} d x
\end{aligned}
$$

The constant depends only on $p$. We also have for $s_{3} \in[-\rho \delta, 0]$ and for $s_{3} \in[L, L+\rho \delta](k=0$ or $N)$

$$
\left|\mathcal{U}^{\prime}\left(s_{3}\right)-A_{k}-B_{k} \wedge M\left(s_{3}\right)\right|^{p}+\delta^{p}\left|\mathcal{R}^{\prime}\left(s_{3}\right)-B_{k}\right|^{p} \leq \frac{C}{\delta^{3}} \int_{\mathcal{P}_{\delta, k}^{\prime}}\left|\left(u-r_{k}\right)(x)\right|^{p} d x .
$$

We integrate on $\left[\alpha_{k}, \alpha_{k+1}\right],-1 \leq k \leq N$. We obtain

$$
\frac{1}{\delta^{p}}\left\|\mathcal{U}^{\prime}-A_{k}-B_{k} \wedge M\right\|_{L^{p}\left(\alpha_{k}, \alpha_{k+1} ; \mathbb{R}^{3}\right)}^{p}+\left\|\mathcal{R}^{\prime}-B_{k}\right\|_{L^{p}\left(\alpha_{k}, \alpha_{k+1} ; \mathbb{R}^{3}\right)}^{p} \leq \frac{C}{\delta^{2}} \mathcal{E}_{p}\left(u, \mathcal{P}_{\delta, k}^{\prime}\right) .
$$

Consequently $\left\|u-U_{e}^{\prime}\right\|_{L^{p}\left(\mathcal{P}_{\delta}^{\prime} ; \mathbb{R}^{3}\right)} \leq C \delta|u|_{\mathcal{E}, \mathcal{P}_{\delta}, p}$, where $C$ depends on the middle line of the rod and on $p$.

Both functions $\mathcal{U}^{\prime}$ and $\mathcal{R}^{\prime}$ belong to $W^{1, p}\left(-\rho \delta, L+\rho \delta ; \mathbb{R}^{3}\right)$. We compute the derivatives of $\mathcal{U}^{\prime}$ and $\mathcal{R}^{\prime}$.

First notice that

$$
\left.\frac{d \mathcal{R}^{\prime}}{d s_{3}}=\frac{d \mathcal{U}^{\prime}}{d s_{3}}-\mathcal{R}^{\prime} \wedge \mathbf{T}=0 \quad \text { on } \quad\right]-\rho \delta, 0[\cup] L, L+\rho \delta[.
$$

We obtain $\left(s_{3} \in[0, L]\right)$

$$
\frac{d \mathcal{U}^{\prime}}{d s_{3}}\left(s_{3}\right)=\frac{1}{\left|B_{1}\right|} \int_{B_{1}} \nabla_{x} u\left(M\left(s_{3}\right)+\delta x\right) \mathbf{T} d x, \quad \frac{d \mathcal{R}^{\prime}}{d s_{3}}\left(s_{3}\right)=\frac{3}{2 M_{1} \delta} \int_{B_{1}} x \wedge \nabla_{x} u\left(M\left(s_{3}\right)+\delta x\right) \mathbf{T} d x .
$$

Hence

$$
\left|\frac{d \mathcal{U}^{\prime}}{d s_{3}}\left(s_{3}\right)-B_{k} \wedge \mathbf{T}\left(s_{3}\right)\right|^{p}+\left|\delta \frac{d \mathcal{R}^{\prime}}{d s_{3}}\left(s_{3}\right)\right|^{p} \leq \frac{C}{\delta^{3}} \int_{\mathcal{P}_{\delta, k}^{\prime}}\left|\nabla_{x}\left(u-r_{k}\right)(x)\right|^{p} d x .
$$

By integrating over $\left[\alpha_{k}, \alpha_{k+1}\right]$ we get

$$
\left\|\frac{d \mathcal{U}^{\prime}}{d s_{3}}-B_{k} \wedge \mathbf{T}\right\|_{L^{p}\left(\alpha_{k}, \alpha_{k+1} ; \mathbb{R}^{3}\right)}^{p}+\delta^{p}\left\|\frac{d \mathcal{R}^{\prime}}{d s_{3}}\right\|_{L^{p}\left(\alpha_{k}, \alpha_{k+1} ; \mathbb{R}^{3}\right)}^{p} \leq \frac{C}{\delta^{2}} \mathcal{E}_{p}\left(u, \mathcal{P}_{\delta, k}^{\prime}\right)
$$


and then we obtain

$$
\left\|\frac{d \mathcal{U}^{\prime}}{d s_{3}}-\mathcal{R}^{\prime} \wedge \mathbf{T}\right\|_{L^{p}\left(0, L ; \mathbb{R}^{3}\right)}+\delta\left\|\frac{d \mathcal{R}^{\prime}}{d s_{3}}\right\|_{L^{p}\left(0, L ; \mathbb{R}^{3}\right)} \leq \frac{C}{\delta^{2 / p}}|u|_{\mathcal{E}, \mathcal{P}_{\delta}^{\prime}, p}
$$

Now we compute the derivatives of $\bar{u}^{\prime}\left(s \in \mathcal{P}_{\delta, k}^{\prime}\right)$

$$
\begin{aligned}
\frac{\partial \bar{u}^{\prime}}{\partial s_{\alpha}} & =\frac{\partial u}{\partial s_{\alpha}}-\mathcal{R}^{\prime} \wedge \mathbf{N}_{\alpha}=\left[\nabla_{x} u \mathbf{N}_{\alpha}-B_{k} \wedge \mathbf{N}_{\alpha}\right]-\left[\mathcal{R}^{\prime}-B_{k}\right] \wedge \mathbf{N}_{\alpha} \\
\frac{\partial \bar{u}^{\prime}}{\partial s_{3}} & =\frac{\partial u}{\partial s_{3}}-\frac{d \mathcal{U}^{\prime}}{d s_{3}}-\frac{d \mathcal{R}^{\prime}}{d s_{3}} \wedge\left(s_{1} \mathbf{N}_{1}+s_{2} \mathbf{N}_{2}\right)-\mathcal{R}^{\prime} \wedge\left(s_{1} \frac{d \mathbf{N}_{1}}{d s_{3}}+s_{2} \frac{d \mathbf{N}_{2}}{d s_{3}}\right) \\
& =\left[\nabla_{x} u\left(\mathbf{T}+s_{1} \frac{d \mathbf{N}_{1}}{d s_{3}}+s_{2} \frac{d \mathbf{N}_{2}}{d s_{3}}\right)-B_{k} \wedge\left(\mathbf{T}+s_{1} \frac{d \mathbf{N}_{1}}{d s_{3}}+s_{2} \frac{d \mathbf{N}_{2}}{d s_{3}}\right)\right]-\left[\frac{d \mathcal{U}^{\prime}}{d s_{3}}-B_{k} \wedge \mathbf{T}\right] \\
& -\frac{d \mathcal{R}^{\prime}}{d s_{3}} \wedge\left(s_{1} \mathbf{N}_{1}+s_{2} \mathbf{N}_{2}\right)-\left(\mathcal{R}^{\prime}-B_{k}\right) \wedge\left(s_{1} \frac{d \mathbf{N}_{1}}{d s_{3}}+s_{2} \frac{d \mathbf{N}_{2}}{d s_{3}}\right)
\end{aligned}
$$

Eventually $\left|\bar{u}^{\prime}\right|_{\mathcal{D}, \mathcal{P}_{\delta}^{\prime}, p} \leq C|u|_{\mathcal{E}, \mathcal{P}_{\delta}, p}$.

Now we modify the elementary displacement $U_{e}^{\prime}$ in order to obtain a new elementary displacement which is rigid in the neighborhood of the extremities of the curved rod.

Let $m$ be an even function $m$ belonging to $\mathcal{C}^{\infty}(\mathbb{R} ;[0,1])$, which satisfies

$$
m(t)=0 \quad \forall t \in[0, \rho], \quad m(t)=1 \quad \forall t \in\left[\rho+1,+\infty\left[, \quad\left|m^{\prime}(t)\right| \leq 2 \quad \forall t \in \mathbb{R} .\right.\right.
$$

The new elementary displacement $U_{e}^{\prime \prime}$ is defined by

$$
\left\{\begin{aligned}
\mathcal{U}^{\prime \prime}\left(s_{3}\right)= & m\left(\frac{s_{3}}{\delta}\right) m\left(\frac{s_{3}-L}{\delta}\right) \mathcal{U}^{\prime}\left(s_{3}\right)+\left(1-m\left(\frac{s_{3}}{\delta}\right)\right)\left[\mathcal{U}^{\prime}(0)+\mathcal{R}^{\prime}(0) \wedge\left(M\left(s_{3}\right)-M(0)\right)\right] \\
& +\left(1-m\left(\frac{s_{3}-L}{\delta}\right)\right)\left[\mathcal{U}^{\prime}(L)+\mathcal{R}^{\prime}(L) \wedge\left(M\left(s_{3}\right)-M(L)\right)\right] \\
\mathcal{R}^{\prime \prime}\left(s_{3}\right)= & m\left(\frac{s_{3}}{\delta}\right) m\left(\frac{s_{3}-L}{\delta}\right) \mathcal{R}^{\prime}\left(s_{3}\right)+\left(1-m\left(\frac{s_{3}}{\delta}\right)\right) \mathcal{R}^{\prime}(0)+\left(1-m\left(\frac{s_{3}-L}{\delta}\right)\right) \mathcal{R}^{\prime}(L) \\
U_{e}^{\prime \prime}(s)= & \left.\mathcal{U}^{\prime \prime}\left(s_{3}\right)+\mathcal{R}^{\prime \prime}\left(s_{3}\right) \wedge\left(s_{1} \mathbf{N}_{1}\left(s_{3}\right)+s_{2} \mathbf{N}_{2}\left(s_{3}\right)\right) \quad s \in\right]-\rho \delta, L+\rho \delta\left[\times \omega_{\delta}\right.
\end{aligned}\right.
$$

We have

$$
U_{e}^{\prime \prime}=U_{e}^{\prime} \quad \text { in } \quad \Phi(](\rho+1) \delta, L-(\rho+1) \delta\left[\times \omega_{\delta}\right)
$$

and $U_{e}^{\prime \prime}$ is a rigid body displacement in the portions $\Phi(]-\rho \delta, \rho \delta\left[\times \omega_{\delta}\right)$ and $\Phi(] L-\rho \delta, L+\rho \delta\left[\times \omega_{\delta}\right)$.

We write

$$
u=U_{e}^{\prime \prime}+\bar{u}^{\prime \prime}
$$

Proposition 2.4 . Let $u \in W^{1, p}\left(\mathcal{P}_{\delta} ; \mathbb{R}^{3}\right), 1<p<\infty$, and $u=U_{e}^{\prime \prime}+\bar{u}^{\prime \prime}$ the decomposition of the extension of $u$ given by (2.11)-(2.12). There exists $\left.\left.\delta_{1}^{\prime \prime} \in\right] 0, \delta_{0}\right]$ wich depends only on $\omega, \rho$ and on the middle line of the rod such that for any $\delta \leq \delta_{1}^{\prime \prime}$ the following estimates hold:

$$
\left\{\begin{array}{l}
\left|\bar{u}^{\prime \prime}\right|_{\mathcal{D}, \mathcal{P}_{\delta}^{\prime}, p} \leq C|u|_{\mathcal{E}, \mathcal{P}_{\delta}, p}, \quad\left\|\bar{u}^{\prime \prime}\right\|_{L^{p}\left(\mathcal{P}_{\delta}^{\prime} ; \mathbb{R}^{3}\right)} \leq C \delta|u|_{\mathcal{E}, \mathcal{P}_{\delta}, p}, \\
\delta\left\|\frac{d \mathcal{R}^{\prime \prime}}{d s_{3}}\right\|_{L^{p}\left(0, L ; \mathbb{R}^{3}\right)}+\left\|\frac{d \mathcal{U}^{\prime \prime}}{d s_{3}}-\mathcal{R}^{\prime \prime} \wedge \mathbf{T}\right\|_{L^{p}\left(0, L ; \mathbb{R}^{3}\right)} \leq \frac{C}{\delta^{2 / p}}|u|_{\mathcal{E}, \mathcal{P}_{\delta}, p}
\end{array}\right.
$$


The constants are independent of $\delta$.

Proof. We easily verify that

$$
\begin{aligned}
&\left\|\mathcal{R}^{\prime \prime}-\mathcal{R}^{\prime}\right\|_{L^{p}\left(0, L ; \mathbb{R}^{3}\right)}+\delta\left\|\frac{d \mathcal{R}^{\prime \prime}}{d s_{3}}-\frac{d \mathcal{R}^{\prime}}{d s_{3}}\right\|_{L^{p}\left(0, L ; \mathbb{R}^{3}\right)} \leq \frac{C}{\delta^{2 / p}}|u|_{\mathcal{E}, \mathcal{P}_{\delta}, p} \\
& \frac{1}{\delta}\left\|\mathcal{U}^{\prime \prime}-\mathcal{U}^{\prime}\right\|_{L^{p}\left(0, L ; \mathbb{R}^{3}\right)}+\left\|\frac{d \mathcal{U}^{\prime \prime}}{d s_{3}}-\frac{d \mathcal{U}^{\prime}}{d s_{3}}\right\|_{L^{p}\left(0, L ; \mathbb{R}^{3}\right)} \leq \frac{C}{\delta^{2 / p}}|u|_{\mathcal{E}, \mathcal{P}_{\delta}, p}
\end{aligned}
$$

The constants depend on $\rho$. Hence we deduce

$$
\left|U_{e}^{\prime \prime}-U_{e}^{\prime}\right|_{\mathcal{D}, \mathcal{P}_{\delta}^{\prime}, p} \leq C|u|_{\mathcal{E}, \mathcal{P}_{\delta}, p} \quad \| U_{e}^{\prime \prime}-\left.U_{e}^{\prime}\right|_{L^{p}\left(\mathcal{P}_{\delta}^{\prime} ; \mathbb{R}^{\prime \prime}\right)} \leq C \delta|u|_{\mathcal{E}, \mathcal{P}_{\delta}, p}
$$

Then from (2.10) we obtain the estimates (2.13).

\section{Decompositions of the displacements of a shell}

\section{Notation}

Let $\omega$ be a bounded domain in $\mathbb{R}^{2}$ with lipschitzian boundary. We give us an injective mapping $\phi$ from $\bar{\omega}$ into $\mathbb{R}^{3}$ of class $\mathcal{C}^{2}$ and we denote $S$ the surface $\phi(\bar{\omega})$. We suppose that the two vectors $\frac{\partial \phi}{\partial s_{\alpha}}(\widehat{s})$ are linearly independent at each point $\widehat{s} \in \bar{\omega}$.

We denote

$$
\mathbf{T}_{1}=\frac{\partial \phi}{\partial s_{1}}, \quad \mathbf{T}_{2}=\frac{\partial \phi}{\partial s_{2}}, \quad \mathbf{N}=\frac{\mathbf{T}_{1} \wedge \mathbf{T}_{2}}{\left\|\mathbf{T}_{1} \wedge \mathbf{T}_{2}\right\|_{2}}
$$

Vectors $\mathbf{T}_{1}$ and $\mathbf{T}_{2}$ are tangential vectors to the surface $S$ and vector $\mathbf{N}$ is a unit normal vector to this surface.

Now we consider the mapping $\Phi: \bar{\omega} \times \mathbb{R} \longrightarrow \mathbb{R}^{3}$

$$
s=\left(s_{1}, s_{2}, s_{3}\right) \longmapsto \phi\left(s_{1}, s_{2}\right)+s_{3} \mathbf{N}\left(s_{1}, s_{2}\right)
$$

There exists $\delta_{0}>0$ depending only on $S$, such that the restriction of $\Phi$ to the compact set $\bar{\Omega}_{\delta_{0}}=$ $\bar{\omega} \times\left[-\delta_{0}, \delta_{0}\right]$ is a $\mathcal{C}^{1}-$ diffeomorphism of that set onto its range. We also choose $\delta_{0}$ such that there exist constants $c_{0}>0$ and $c_{1} \geq c_{0}$ verifying

$$
\forall s \in \Omega_{\delta_{0}}, \quad c_{0} \leq \mid\|\nabla \Phi(s)\|_{2} \leq c_{1}
$$

These constants depend only on $\phi$.

Definition 5 . The shell $\mathcal{Q}_{\delta}$ is defined as follows :

$$
\left.\left.\left.\mathcal{Q}_{\delta}=\Phi\left(\Omega_{\delta}\right) \quad \text { for } \delta \in\right] 0, \delta_{0}\right], \quad \Omega_{\delta}=\omega \times\right]-\delta, \delta[.
$$

The mid-surface of the shell is $S$. The lateral boundary of the shell is $\Gamma_{\delta}=\Phi(\partial \omega \times]-\delta, \delta[)$.

Notation (i). Reference domains and running points. We denote $x$ the running point of $\mathcal{Q}_{\delta}$. The running point of the domain $\Omega_{\delta}$ is $s$.

(ii). Displacements. For any displacement $u \in L^{1}\left(\mathcal{Q}_{\delta} ; \mathbb{R}^{3}\right)$, we write $u$ instead of $u \circ \Phi$. 


\section{First decomposition of displacements of a shell.}

Definition 6 . We call elementary displacement of a shell, any element $v$ of $L^{1}\left(\Omega_{\delta} ; \mathbb{R}^{3}\right)$ that is written in the form

$$
v(s)=\mathcal{V}(\widehat{s})+s_{3} \mathcal{A}(\widehat{s}) \wedge \mathbf{N}(\widehat{s}), \quad s=\left(s_{1}, s_{2}, s_{3}\right)=\left(\widehat{s}, s_{3}\right) \in \Omega_{\delta}
$$

where $\mathcal{V}$ and $\mathcal{A}$ are functions in $L^{1}\left(\omega ; \mathbb{R}^{3}\right)$.

The first component $\mathcal{V}$ of $v$ is the displacement of the middle surface. The second component $\mathcal{A}$, gives us an information about the relative displacement of the fibers $\{M(\widehat{s})\} \times]-\delta, \delta$ [ of the shell, that is to say, rotations whose axis are directed along the vector $\mathcal{A}(\widehat{s})$.

To any displacement $u$ of the rod we associate an elementary displacement defined as follows:

Definition 7 . The elementary displacement $U_{e}$, associated to $u \in L^{1}\left(\mathcal{Q}_{\delta} ; \mathbb{R}^{3}\right)$, is given by

$$
\left.U_{e}\left(., s_{3}\right)=\mathcal{U}+s_{3} \mathcal{R} \wedge \mathbf{N}, \quad s_{3} \in\right]-\delta, \delta[,
$$

where

$$
\mathcal{U}=\frac{1}{2 \delta} \int_{-\delta}^{\delta} u\left(., s_{3}\right) d s_{3}, \quad \mathcal{R}=\frac{3}{2 \delta^{3}} \int_{-\delta}^{\delta} s_{3} \mathbf{N}(.) \wedge u\left(., s_{3}\right) d s_{3}
$$

We write

$$
u=U_{e}+\bar{u}
$$

This displacement $\bar{u}$ is the warping.

The next theorem plays a fundamental role in the studies of plates and shells with the unfolding method. It provides sharp estimates of displacements $U_{e}$ and $\bar{u}$ in terms of $\delta$ and of the strain energy of $u$.

Theorem 3.1. Let $u \in W^{1, p}\left(\mathcal{Q}_{\delta} ; \mathbb{R}^{3}\right), 1<p<\infty$, and $U_{e}$ be the elementary displacement defined by (3.1)-(3.3). There exists $\left.\left.\delta_{1} \in\right] 0, \delta_{0}\right]$ which depends only on the middle surface of the shell such that for any $\delta \leq \delta_{1}$ the following estimates hold:

$$
\left|U_{e}\right|_{\mathcal{E}, \mathcal{Q}_{\delta}, p}+|\bar{u}|_{\mathcal{D}, \mathcal{Q}_{\delta}, p} \leq C|u|_{\mathcal{E}, \mathcal{Q}_{\delta}, p}, \quad\|\bar{u}\|_{L^{p}\left(\mathcal{Q}_{\delta} ; \mathbb{R}^{3}\right)} \leq C \delta|u|_{\mathcal{E}, \mathcal{Q}_{\delta}, p}
$$

The constants are independent of $\delta$.

Proof .

Step 1 . We prove that there exist $R \geq 1$ depending only on $\partial \omega$ and a family of open sets $\left(\omega_{\delta, k}\right)_{k \in \mathcal{N}_{\delta}}$ verifying

$$
\bar{\omega}=\bigcup_{k \in \mathcal{N}_{\delta}} \bar{\omega}_{\delta, k}
$$

and such that every open set of this family has a diameter less than $R \delta$ and is star-shaped with respect to a disc of radius $\delta / 2$ and moreover such that any point of $\omega$ belongs to a finite number (independent of $\delta$ ) of sets $\bar{\omega}_{\delta, k}$.

The boundary of $\omega$ is lipschitzian. Hence there exist constants $C<B<A$ and $M$ strictly positive, a finite number $K$ of local coordinate systems $\left(s_{1 r}, s_{2 r}\right)$ in $\left(O_{r} ; \mathbf{e}_{1 r}, \mathbf{e}_{2 r}\right)$ and mappings $f_{r}:[0, A] \longrightarrow \mathbb{R}$, 
Lipschitz continuous with ratio $M, 1 \leq r \leq K$, such that

$$
\left\{\begin{array}{l}
\partial \omega=\bigcup_{r=1}^{K}\left\{\left(s_{1 r}, s_{2 r}\right) \mid s_{2 r}=f_{r}\left(s_{1 r}\right), \quad s_{1 r} \in\right] B / 2, A-B / 2[\} \\
\{\widehat{s} \in \omega \mid \operatorname{dist}(\widehat{s}, \partial \omega)<C\} \subset \bigcup_{r=1}^{K}\left\{\left(s_{1 r}, s_{2 r}\right) \mid f_{r}\left(s_{1 r}\right)-B<s_{2 r}<f_{r}\left(x_{1 r}\right), s_{1 r} \in\right] 0, A[\} \subset \omega \\
\omega_{C} \backslash \omega \subset \bigcup_{r=1}^{K}\left\{\left(s_{1 r}, s_{2 r}\right) \mid f_{r}\left(s_{1 r}\right)<s_{2 r}<f_{r}\left(s_{1 r}\right)+B, s_{1 r} \in\right] 0, A[\} \subset \mathbb{R}^{2} \backslash \omega
\end{array}\right.
$$

Through the use of easy geometrical arguments we show that if $\delta \leq \inf \{C / 2, B / 4(M+1)\}$, we have

$$
\{\widehat{s} \in \omega \mid \operatorname{dist}(\widehat{s}, \partial \omega)<2 \delta\} \subset \bigcup_{r=1}^{K}\left\{\left(s_{1 r}, s_{2 r}\right) \mid f_{r}\left(s_{1 r}\right)-2 \delta(1+M)<s_{2 r}<f_{r}\left(s_{1 r}\right), s_{1 r} \in\right] 0, A[\} .
$$

We suppose also that $\delta \leq \inf \{A / 6, B /(6 M+2)\}$. Let $N$ be an integer belonging to $\left[\frac{2}{3} \frac{A}{2 \delta}, \frac{A}{2 \delta}\right]$, and set $\alpha_{k}=k \frac{A}{2 N}, k \in\{0, \ldots, 2 N\}$. We have $N$ greater than 3 and $\delta \leq \frac{A}{2 N} \leq \frac{3}{2} \delta$. For any $k \in\{0, \ldots, N-1\}$ the domains

$$
\mathcal{B}_{\delta, k}^{(r)}=\left\{\left(s_{1 r}, s_{2 r}\right) \mid f_{r}\left(s_{1}\right)-2 \delta(3 M+1)<s_{2 r}<f_{r}\left(s_{1 r}\right), s_{1 r} \in\right] \alpha_{2 k}, \alpha_{2(k+1)}[\}
$$

are star-shaped with respect to the disc centered at $\left(\alpha_{2 k+1}, f_{r}\left(\alpha_{2 k+1}\right)-(3 M+1) \delta\right)$ and of radius $\delta / 2$ and they have a diameter less than $(8 M+5) \delta=R \delta$.

Now we complete the family of sets $\mathcal{B}_{\delta, k}^{(r)}, r \in\{1, \ldots, K\}$ and $k \in\{0, \ldots, N-1\}$, by taking all domains

$$
] k \delta,(k+1) \delta[\times] l \delta,(l+1) \delta\left[, \quad(k, l) \in \mathbb{Z}^{2}\right.
$$

included in $\omega$. We denote $\left(\omega_{\delta, k}\right)_{k \in \mathcal{N}_{\delta}}$ the family of all these sets.

\section{Step 2 .}

The open set $\left.\Omega_{\delta, k}=\omega_{\delta, k} \times\right]-\delta, \delta[$ has a diameter less than $2 R \delta$ and is star-shaped with respect to a ball centered on $\omega$ and of radius $\delta / 2$ (here $R_{1}=1 / 2$ ). The mapping $\widehat{s} \longmapsto\left(\mathbf{T}_{1}, \mathbf{T}_{2}, \mathbf{N}\right)$ belongs to $\mathcal{C}^{1}\left(\bar{\omega} ;\left(\mathbb{R}^{3}\right)^{3}\right)$. Hence, there exists $C_{0}>0$ which does not depend on $\delta$ such that

$$
\forall s \in \Omega_{\delta, k}, \quad \forall \tilde{s} \in \Omega_{\delta, k}, \quad\|\| \nabla \Phi(s)-\nabla \Phi(\tilde{s}) \|_{2} \leq C_{0} \delta
$$

In the sequel we will work with the portions $\mathcal{Q}_{\delta, k}=\Phi\left(\Omega_{\delta, k}\right), k \in \mathcal{N}_{\delta}$, of the shell.

Now we take $\delta$ such that

$$
\delta \leq \inf \left\{\frac{c_{0}^{3}}{C_{0} c_{1}^{2}}\left(\frac{1 / 2}{4(2 R)}\right)^{3}, \delta_{0}\right\}
$$

Then we have

$$
C_{0} \delta \leq\left(\frac{1 / 2}{4(2 R)}\right)^{3} \inf _{s \in \Omega_{\delta}}\left\{\left\|\left|\nabla \Phi(s)\left\|\left.\right|_{2} ^{3}\right\|\right|(\nabla \Phi(s))^{-1}\right\|_{2}^{2}\right\}
$$

Thanks to corollary of Lemmas 4.1 and 4.2 (see Annexes) the domain $\mathcal{Q}_{\delta, k}$ has a diameter less than $6 R \delta$ and is star-shaped with respect to a ball of radius $\delta / 16$. 
From Theorem 1.3 there exist rigid body displacements $r_{k}$ such that

$$
\mathcal{D}_{p}\left(u-r_{k}, \mathcal{Q}_{\delta, k}\right) \leq C \mathcal{E}_{p}\left(u, \mathcal{Q}_{\delta, k}\right), \quad\left\|u-r_{k}\right\|_{L^{p}\left(\mathcal{Q}_{\delta, k} ; \mathbb{R}^{3}\right)}^{p} \leq C \delta^{p} \mathcal{E}_{p}\left(u, \mathcal{Q}_{\delta, k}\right),
$$

where the constants do not depend on $k$ and $\delta$.

Now we go on as in Theorem 2.1. Recall that the displacements $r_{k}$ are of the form

$$
r_{k}(x)=A_{k}+B_{k} \wedge x, \quad x \in \mathcal{Q}_{\delta, k}, \quad A_{k}, B_{k} \in \mathbb{R}^{3} .
$$

Taking the mean value on the fibers of the shell, and using definition (3.2) of $\mathcal{U}$ and $\mathcal{R}$, we deduce the inequalities

$$
\left\{\begin{array}{l}
\left\|\mathcal{U}-A_{k}-B_{k} \wedge \phi\right\|_{L^{p}\left(\omega_{\delta, k} ; \mathbb{R}^{3}\right)}^{p} \leq C \delta^{p-1} \mathcal{E}_{p}\left(u, \mathcal{Q}_{\delta, k}\right) \\
\left\|\mathcal{R} \wedge \mathbf{N}-B_{k} \wedge \mathbf{N}\right\|_{L^{p}\left(\omega_{\delta, k} ; \mathbb{R}^{3}\right)}^{p} \leq \frac{C}{\delta} \mathcal{E}_{p}\left(u, \mathcal{Q}_{\delta, k}\right)
\end{array}\right.
$$

where the constants are independent of $k$ and $\delta$. Consequently $\left\|u-U_{e}\right\|_{L^{p}\left(\Omega_{\delta} ; \mathbb{R}^{3}\right)} \leq C \delta|u|_{\mathcal{E}, \mathcal{Q}_{\delta}, p}$, where $C$ depends only on the middle surface of the shell and on $p$.

Note now that both functions $\mathcal{U}$ and $\mathcal{R}$ belong to $W^{1, p}\left(\omega ; \mathbb{R}^{3}\right)$. We compute the derivatives of the displacement $u$ to get

$$
\frac{\partial u}{\partial s_{1}}=\nabla_{x} u\left(\mathbf{T}_{1}+s_{3} \frac{\partial \mathbf{N}}{\partial s_{1}}\right), \quad \frac{\partial u}{\partial s_{2}}=\nabla_{x} u\left(\mathbf{T}_{2}+s_{3} \frac{\partial \mathbf{N}}{\partial s_{2}}\right), \quad \frac{\partial u}{\partial s_{3}}=\nabla_{x} u \mathbf{N} .
$$

Taking the restrictions of these derivatives to $\Omega_{\delta, k}$, from (3.5) we have the estimates

$$
\left\|\frac{\partial u}{\partial s_{\alpha}}-B_{k} \wedge\left(\mathbf{T}_{\alpha}+s_{3} \frac{\partial \mathbf{N}}{\partial s_{\alpha}}\right)\right\|_{L^{p}\left(\Omega_{\delta, k} ; \mathbb{R}^{3}\right)}^{p}+\left\|\frac{\partial u}{\partial s_{3}}-B_{k} \wedge \mathbf{N}\right\|_{L^{p}\left(\Omega_{\delta, k} ; \mathbb{R}^{3}\right)}^{p} \leq C \mathcal{E}_{p}\left(u, \mathcal{Q}_{\delta, k}\right) .
$$

By taking the mean value of $\frac{\partial u}{\partial s_{\alpha}}-B_{k} \wedge\left(\mathbf{T}_{\alpha}+s_{3} \frac{\partial \mathbf{N}}{\partial s_{\alpha}}\right)$ on the fibers we get

$$
\left\|\frac{\partial \mathcal{U}}{\partial s_{\alpha}}-B_{k} \wedge \mathbf{T}_{\alpha}\right\|_{L^{p}\left(\omega_{\delta, k} ; \mathbb{R}^{3}\right)}^{p} \leq \frac{C}{\delta} \mathcal{E}_{p}\left(u, \Omega_{\delta, k}\right)
$$

We now estimate the $L^{p}$ norm of the derivatives of $\mathcal{R} \wedge \mathbf{N}$ with respect to $s_{\alpha}$

$$
\begin{aligned}
\mathcal{R} & \wedge \mathbf{N}=\frac{3}{2 \delta^{3}} \int_{-\delta}^{\delta} s_{3}\left[u\left(., s_{3}\right)-\left(u\left(., s_{3}\right) \cdot \mathbf{N}\right) \mathbf{N}\right] d s_{3} \\
\frac{\partial}{\partial s_{\alpha}}(\mathcal{R} & \wedge \mathbf{N})=\frac{3}{2 \delta^{3}} \int_{-\delta}^{\delta} s_{3}\left[\frac{\partial u}{\partial s_{\alpha}}\left(., s_{3}\right)-\left(\frac{\partial u}{\partial s_{\alpha}}\left(., s_{3}\right) \cdot \mathbf{N}\right) \mathbf{N}-\left(u\left(., s_{3}\right) \cdot \frac{\partial \mathbf{N}}{\partial s_{\alpha}}\right) \mathbf{N}-\left(u\left(., s_{3}\right) \cdot \mathbf{N}\right) \frac{\partial \mathbf{N}}{\partial s_{\alpha}}\right] d s_{3}
\end{aligned}
$$

Then we replace $\frac{\partial u}{\partial s_{\alpha}}$ by $B_{k} \wedge\left(\mathbf{T}_{\alpha}+s_{3} \frac{\partial \mathbf{N}}{\partial s_{\alpha}}\right)$ and $u$ by $A_{k}+B_{k} \wedge\left(\phi+s_{3} \mathbf{N}\right)$. We obtain

$$
\left\|\frac{\partial}{\partial s_{\alpha}}(\mathcal{R} \wedge \mathbf{N})-B_{k} \wedge \frac{\partial \mathbf{N}}{\partial s_{\alpha}}\right\|_{L^{p}\left(\omega_{\delta, k} ; \mathbb{R}^{3}\right)}^{p} \leq \frac{C}{\delta^{p+1}} \mathcal{E}_{p}\left(u, \Omega_{\delta, k}\right)
$$

From the above inequalities and from (3.6) we get $\left\|\frac{\partial \bar{u}}{\partial s_{i}}\right\|_{L^{p}\left(\omega_{\delta, k} ; \mathbb{R}^{3}\right)}^{p} \leq C \mathcal{E}_{p}\left(u, \Omega_{\delta, k}\right)$, hence with a change of variables $|\bar{u}|_{\mathcal{D}, \mathcal{Q}_{\delta}, p} \leq C|u|_{\mathcal{E}, \mathcal{Q}_{\delta}, p}$. Eventually observe that

$$
\left|U_{e}\right|_{\mathcal{E}, \mathcal{Q}_{\delta}, p} \leq\left|U_{e}+\bar{u}\right|_{\mathcal{E}, \mathcal{Q}_{\delta}, p}+|\bar{u}|_{\mathcal{E}, \mathcal{Q}_{\delta}, p} \leq|u|_{\mathcal{E}, \mathcal{Q}_{\delta}, p}+|\bar{u}|_{\mathcal{D}, \mathcal{Q}_{\delta}, p} \leq C|u|_{\mathcal{E}, \mathcal{Q}_{\delta}, p}
$$


where the constant does not depend on $\delta$.

Remark . Thanks to (3.7) and (3.6) it follows by eliminating $B_{k}$

$$
\left\|\frac{\partial \mathcal{U}}{\partial s_{\alpha}} \cdot \mathbf{T}_{\beta}+\frac{\partial \mathcal{U}}{\partial s_{\beta}} \cdot \mathbf{T}_{\alpha}\right\|_{L^{p}(\omega)}+\left\|\frac{\partial \mathcal{U}}{\partial s_{\alpha}} \cdot \mathbf{N}+(\mathcal{R} \wedge \mathbf{N}) \cdot \mathbf{T}_{\alpha}\right\|_{L^{p}(\omega)} \leq \frac{C}{\delta^{1 / p}}|u|_{\mathcal{E}, \mathcal{Q}_{\delta}, p} .
$$

Now from (3.7) and (3.8) we obtain by eliminating $B_{k}$

$$
\left\|\frac{\partial}{\partial s_{\alpha}}(\mathcal{R} \wedge \mathbf{N}) \cdot \mathbf{T}_{\beta}+\frac{\partial \mathcal{U}}{\partial s_{\beta}} \cdot \frac{\partial \mathbf{N}}{\partial s_{\alpha}}\right\|_{L^{p}(\omega)} \leq \frac{C}{\delta^{1+1 / p}}|u|_{\mathcal{E}, \mathcal{Q}_{\delta}, p} .
$$

The constants do not depend on $\delta$. The inequality $\left|U_{e}\right|_{\mathcal{E}, \mathcal{Q}_{\delta}, p} \leq C|u|_{\mathcal{E}, \mathcal{Q}_{\delta}, p}$ is equivalent to the above estimates (3.9) and (3.10) (see [6] for the expressions of the covariant components of the strain tensor).

\section{Second decomposition of displacements of a shell.}

For any $\eta>0$ we denote $\omega_{\eta}$ the open set

$$
\omega_{\eta}=\left\{\left(s_{1}, s_{2}\right) \in \mathbb{R}^{2} \mid \operatorname{dist}\left(\left(s_{1}, s_{2}\right), \omega\right)<\eta\right\}
$$

In this paragraph we suppose that the mapping $\Phi$ introduced in the previous subsection is defined on $\bar{\omega}_{l} \times\left[-\delta_{0}, \delta_{0}\right], l>0$, and it is a $\mathcal{C}^{1}-$ diffeomorphism of that set onto its range and allways verifies

$$
\forall s \in \bar{\omega}_{l} \times\left[-\delta_{0}, \delta_{0}\right], \quad c_{0} \leq\|\| \nabla \Phi(s) \|_{2} \leq c_{1}
$$

Lemma 3.2 . Let $u$ be a displacement in $W^{1, p}\left(\mathcal{Q}_{\delta} ; \mathbb{R}^{3}\right), 1<p<\infty$. There exists an extension $P(u)$ of $u$ to the shell

$$
\mathcal{Q}_{\delta}^{\prime}=\Phi\left(\omega_{2 \delta} \times\right]-\delta, \delta[)
$$

such that

$$
P(u) \in W^{1, p}\left(\mathcal{Q}_{\delta}^{\prime} ; \mathbb{R}^{3}\right) \quad P(u)_{\left.\right|_{\mathcal{Q}_{\delta}}}=u \quad|P(u)|_{\mathcal{E}, \mathcal{Q}_{\delta}^{\prime}, p} \leq C|u|_{\mathcal{E}, \mathcal{Q}_{\delta}, p} .
$$

The constant does not depend of $\delta$ and of $u$.

Proof . See Section V.

From now on any displacement belonging to $W^{1, p}\left(\mathcal{Q}_{\delta} ; \mathbb{R}^{3}\right)$ is extended to a displacement belonging to $W^{1, p}\left(\mathcal{Q}_{\delta}^{\prime} ; \mathbb{R}^{3}\right)$. Again we denote $u$ this extension.

Let $B$ be the ball centered at the origin and of radius $1 / 16$. We put

$$
|B|=\int_{B} d x \quad M=\int_{B}\|x\|_{2}^{2} d x
$$

To any displacement $u$ of the shell we associate a new elementary displacement defined as follows :

Definition 8 . The elementary displacement $U_{e}^{\prime}$, associated to $u \in L^{1}\left(\mathcal{Q}_{\delta}^{\prime} ; \mathbb{R}^{3}\right)$, is given by

$$
\left.U_{e}^{\prime}\left(., s_{3}\right)=\mathcal{U}^{\prime}+s_{3} \mathcal{R}^{\prime} \wedge \mathbf{N}, \quad s_{3} \in\right]-\delta, \delta[,
$$

where

$$
\mathcal{U}^{\prime}(\widehat{s})=\frac{1}{|B|} \int_{B} u(\phi(\widehat{s})+\delta x) d x, \quad \mathcal{R}^{\prime}(\widehat{s})=\frac{3}{2 M \delta} \int_{B} x \wedge u(\phi(\widehat{s})+\delta x) d x \quad \widehat{s} \in \omega
$$


We write

$$
u=U_{e}^{\prime}+\bar{u}^{\prime}
$$

Theorem 3.3 . Let $u \in W^{1, p}\left(\mathcal{Q}_{\delta} ; \mathbb{R}^{3}\right), 1<p<\infty$, and $u=U_{e}^{\prime}+\bar{u}^{\prime}$ be the decomposition of $u$ given by (3.11)-(3.13). There exists $\left.\left.\delta_{1}^{\prime} \in\right] 0, \delta_{0}\right]$ which depends only on the middle surface of the shell such that for any $\delta \leq \delta_{1}^{\prime}$ the following estimates hold:

$$
\left\{\begin{array}{l}
\left|\bar{u}^{\prime}\right|_{\mathcal{D}, \mathcal{Q}_{\delta}, p} \leq C|u|_{\mathcal{E}, \mathcal{Q}_{\delta}, p}, \quad \quad\left\|\bar{u}^{\prime}\right\|_{L^{p}\left(\mathcal{Q}_{\delta} ; \mathbb{R}^{3}\right)} \leq C \delta|u|_{\mathcal{E}, \mathcal{Q}_{\delta}, p}, \\
\left\|\frac{\partial \mathcal{U}^{\prime}}{\partial s_{\alpha}}-\mathcal{R}^{\prime} \wedge \mathbf{T}_{\alpha}\right\|_{L^{p}\left(\omega ; \mathbb{R}^{3}\right)}+\delta\left\|\frac{\partial \mathcal{R}^{\prime}}{\partial s_{\alpha}}\right\|_{L^{p}\left(\omega ; \mathbb{R}^{3}\right)} \leq \frac{C}{\delta^{1 / p}}|u|_{\mathcal{E}, \mathcal{Q}_{\delta}, p} .
\end{array}\right.
$$

The constants are independent of $\delta$.

Proof . Let $\mathcal{N}_{\delta}$ be the set of every $(k, l) \in \mathbb{Z}^{2}$ such that the open set

$$
\left.\omega_{\delta, k l}^{\prime}=\right](k-1 / 3) \delta,(k+4 / 3) \delta[\times](l-1 / 3) \delta,(l+4 / 3) \delta[
$$

is included in $\omega_{2 \delta}$. We put

$$
\left.\omega_{\delta, k l}=\right] k \delta,(k+1) \delta[\times] l \delta,(l+1) \delta[
$$

We have

$$
\omega \subset \bigcup_{(k, l) \in \mathcal{N}_{\delta}} \bar{\omega}_{\delta, k l}
$$

The open set $\left.\Omega_{\delta, k l}^{\prime}=\omega_{\delta, k l}^{\prime} \times\right]-\delta, \delta[$ has a diameter less than $4 \delta$ and it is star-shaped with respect to the ball centered at the point $((k+1 / 2) \delta,(l+1 / 2) \delta)$ belonging to $\omega$ and of radius $\delta / 2$. The mapping $\widehat{s} \longmapsto\left(\mathbf{T}_{1}, \mathbf{T}_{2}, \mathbf{N}\right)$ belongs to $\mathcal{C}^{1}\left(\bar{\omega}_{2 \delta_{0}} ;\left(\mathbb{R}^{3}\right)^{3}\right)$. There exists $C_{0}^{\prime}>0$ which does not depend on $\delta$ such that

$$
\forall(s, \tilde{s}) \in \Omega_{\delta, k l}^{\prime} \times \Omega_{\delta, k l}^{\prime}, \quad \quad\|\nabla \Phi(s)-\nabla \Phi(\tilde{s})\|_{2} \leq C_{0} \delta
$$

In the sequel we will work with the portions $\mathcal{Q}_{\delta, k l}^{\prime}=\Phi\left(\Omega_{\delta, k l}^{\prime}\right),(k, l) \in \mathcal{N}_{\delta}$, of the shell.

Now we take $\delta$ such that

$$
\delta \leq \frac{c_{0}^{3}}{C_{0}^{\prime} c_{1}^{2}}\left(\frac{1 / 2}{4.4}\right)^{3}
$$

then we have

$$
C_{0}^{\prime} \delta \leq\left(\frac{1 / 2}{4.4}\right)^{3} \inf _{s \in \Omega_{\delta}}\left\{\|\nabla \Phi(s)\|_{2}^{3}\|\|(\nabla \Phi(s))^{-1}\|\|_{2}^{2}\right\}
$$

Thanks to corollary of Lemmas 4.1 and 4.2 (see Section V) the domain $\mathcal{Q}_{\delta, k}$ has a diameter less than $12 \delta$ and it is star-shaped with respect to a ball of radius $\delta / 16$.

From Theorem 1.3 there exist rigid body displacements $r_{k l}$ such that

$$
\mathcal{D}_{p}\left(u-r_{k l}, \mathcal{Q}_{\delta, k l}^{\prime}\right) \leq C \mathcal{E}_{p}\left(u, \mathcal{Q}_{\delta, k l}^{\prime}\right), \quad\left\|u-r_{k l}\right\|_{L^{p}\left(\mathcal{Q}_{\delta, k l}^{\prime}, \mathbb{R}^{3}\right)}^{p} \leq C \delta^{p} \mathcal{E}_{p}\left(u, \mathcal{Q}_{\delta, k l}^{\prime}\right)
$$

where the constants do not depend on $k, l$ and $\delta$.

Now we go on as in Theorem 3.1. Recall that the displacements $r_{k l}$ are of the form

$$
r_{k l}(x)=A_{k l}+B_{k l} \wedge x, \quad x \in \mathcal{Q}_{\delta, k l}^{\prime}, \quad A_{k l}, B_{k l} \in \mathbb{R}^{3} .
$$


Using definition (3.11) of $\mathcal{U}^{\prime}$ and $\mathcal{R}^{\prime}$ we obtain

$$
\begin{aligned}
\left|\mathcal{U}^{\prime}(\widehat{s})-A_{k l}-B_{k l} \wedge \phi(\widehat{s})\right|^{p} & \leq \frac{1}{|B|} \int_{B}\left|\left(u-r_{k l}\right)(\phi(\widehat{s})+\delta x)\right|^{p} d x \leq \frac{1}{\delta^{3}|B|} \int_{\mathcal{Q}_{\delta, k l}^{\prime}}\left|\left(u-r_{k l}\right)(x)\right|^{p} d x \\
\left|\mathcal{R}^{\prime}(\widehat{s})-B_{k l}\right|^{p} & \leq \frac{C}{\delta^{p}} \int_{B}\left|\left(u-r_{k l}\right)(\phi(\widehat{s})+\delta x)\right|^{p} d x \leq \frac{C}{\delta^{p+3}} \int_{\mathcal{Q}_{\delta, k l}^{\prime}}\left|\left(u-r_{k l}\right)(x)\right|^{p} d x
\end{aligned}
$$

The constant depends only on $p$. We integrate on $\omega_{\delta, k l}$. Thanks to (3.15) we obtain

$$
\left\|\mathcal{U}^{\prime}-A_{k l}-B_{k l} \wedge \phi\right\|_{L^{p}\left(\omega_{\delta, k l} ; \mathbb{R}^{3}\right)}^{p} \leq C \delta^{p-1} \mathcal{E}_{p}\left(u, \mathcal{Q}_{\delta, k l}^{\prime}\right), \quad\left\|\mathcal{R}^{\prime}-B_{k l}\right\|_{L^{p}\left(\omega_{\delta, k l} ; \mathbb{R}^{3}\right)}^{p} \leq \frac{C}{\delta} \mathcal{E}_{p}\left(u, \mathcal{Q}_{\delta, k l}^{\prime}\right)
$$

Consequently $\left\|u-U_{e}^{\prime}\right\|_{L^{p}\left(\Omega_{\delta} ; \mathbb{R}^{3}\right)} \leq C \delta|u|_{\mathcal{E}, \mathcal{Q}_{\delta}^{\prime}, p}$, where $C$ depends only on the middle surface of the shell and on $p$. Both functions $\mathcal{U}^{\prime}$ and $\mathcal{R}^{\prime}$ belong to $W^{1, p}\left(\omega ; \mathbb{R}^{3}\right)$. We compute the derivatives of $\mathcal{U}^{\prime}$ and $\mathcal{R}^{\prime}$ to get

$$
\frac{\partial \mathcal{U}^{\prime}}{\partial s_{\alpha}}(\widehat{s})=\frac{1}{|B|} \int_{B} \nabla_{x} u(\phi(\widehat{s})+\delta x) \mathbf{T}_{\alpha} d x, \quad \frac{\partial \mathcal{R}^{\prime}}{\partial s_{\alpha}}(\widehat{s})=\frac{3}{2 M \delta} \int_{B} x \wedge \nabla_{x} u(\phi(\widehat{s})+\delta x) \mathbf{T}_{\alpha} d x
$$

Hence

$$
\left|\frac{\partial \mathcal{U}^{\prime}}{\partial s_{\alpha}}(\widehat{s})-B_{k} \wedge \mathbf{T}_{\alpha}\right|^{p}+\delta^{p}\left|\frac{\partial \mathcal{R}^{\prime}}{\partial s_{\alpha}}(\widehat{s})\right|^{p} \leq \frac{C}{\delta^{3}} \int_{\mathcal{Q}_{\delta, k l}^{\prime}}\left|\nabla_{x}\left(u-r_{k l}\right)(x)\right|^{p} d x
$$

By integrating over $\omega_{\delta, k l}$ we obtain

$$
\left\|\frac{\partial \mathcal{U}^{\prime}}{\partial s_{\alpha}}-B_{k} \wedge \mathbf{T}_{\alpha}\right\|_{L^{p}\left(\omega_{\delta, k l} ; \mathbb{R}^{3}\right)}^{p}+\delta^{p}\left\|\frac{\partial \mathcal{R}^{\prime}}{\partial s_{\alpha}}\right\|_{L^{p}\left(\omega_{\delta, k l} ; \mathbb{R}^{3}\right)}^{p} \leq \frac{C}{\delta} \mathcal{E}_{p}\left(u, \mathcal{Q}_{\delta, k l}^{\prime}\right)
$$

Thanks to (3.16) we get

$$
\left\|\frac{\partial \mathcal{U}^{\prime}}{\partial s_{\alpha}}-\mathcal{R}^{\prime} \wedge \mathbf{T}_{\alpha}\right\|_{L^{p}\left(\omega ; \mathbb{R}^{3}\right)}+\delta\left\|\frac{\partial \mathcal{R}^{\prime}}{\partial s_{\alpha}}\right\|_{L^{p}\left(\omega ; \mathbb{R}^{3}\right)} \leq \frac{C}{\delta^{1 / p}}|u|_{\mathcal{E}, \mathcal{Q}_{\delta}^{\prime}, p} .
$$

Now we estimate $\left|\bar{u}^{\prime}\right|_{\mathcal{D}, \mathcal{Q}_{\delta}, p}$. We have

$$
\begin{aligned}
& \frac{\partial \bar{u}^{\prime}}{\partial s_{3}}=\frac{\partial u}{\partial s_{3}}-\mathcal{R}^{\prime} \wedge \mathbf{N}=\left[\nabla_{x} u \mathbf{N}-B_{k l}\right.\wedge \mathbf{N}]-\left[\mathcal{R}^{\prime}-B_{k l}\right] \wedge \mathbf{N} \\
& \frac{\partial \bar{u}^{\prime}}{\partial s_{\alpha}}=\frac{\partial u}{\partial s_{\alpha}}-\frac{\partial \mathcal{U}^{\prime}}{\partial s_{\alpha}}-s_{3} \frac{\partial \mathcal{R}^{\prime}}{\partial s_{\alpha}} \wedge \mathbf{N}-s_{3} \mathcal{R}^{\prime} \wedge \frac{\partial \mathbf{N}}{\partial s_{\alpha}}=\left[\nabla_{x} u\left(\mathbf{T}_{\alpha}+s_{3} \frac{\partial \mathbf{N}}{\partial s_{\alpha}}\right)-B_{k l} \wedge\left(\mathbf{T}_{\alpha}+s_{3} \frac{\partial \mathbf{N}}{\partial s_{\alpha}}\right)\right] \\
&-\left[\frac{\partial \mathcal{U}^{\prime}}{\partial s_{\alpha}}-B_{k l} \wedge \mathbf{T}_{\alpha}\right]-s_{3} \frac{\partial \mathcal{R}^{\prime}}{\partial s_{\alpha}} \wedge \mathbf{N}-s_{3}\left(\mathcal{R}^{\prime}-B_{k l}\right) \wedge \frac{\partial \mathbf{N}}{\partial s_{\alpha}}
\end{aligned}
$$

Thanks to (3.15), (3.16) and (3.17) we obtain $\left\|\frac{\partial \bar{u}^{\prime}}{\partial s_{i}}\right\|_{L^{p}\left(\Omega_{\delta, k l} ; \mathbb{R}^{3}\right)}^{p} \leq C \mathcal{E}_{p}\left(u, \mathcal{Q}_{\delta, k l}^{\prime}\right)$, and eventually $\left|\bar{u}^{\prime}\right|_{\mathcal{D}, \mathcal{Q}_{\delta}, p} \leq$ $C|u|_{\mathcal{E}, \mathcal{Q}_{\delta}^{\prime}, p^{\prime}}$

Now we compare the elementary displacements $U_{e}$ and $U_{e}^{\prime}$.

Corollary 1 . We have

$$
\left|U_{e}-U_{e}^{\prime}\right|_{\mathcal{D}, \mathcal{Q}_{\delta}, p} \leq C|u|_{\mathcal{E}, \mathcal{Q}_{\delta}, p}, \quad \quad\left\|U_{e}-U_{e}^{\prime}\right\|_{L^{p}\left(\mathcal{Q}_{\delta} ; \mathbb{R}^{3}\right)} \leq C \delta|u|_{\mathcal{E}, \mathcal{Q}_{\delta}, p}
$$


Moreover

$$
\begin{cases}\left\|\mathcal{U}^{\prime}-\mathcal{U}\right\|_{L^{p}\left(\omega ; \mathbb{R}^{3}\right)} \leq C \delta^{1-1 / p}|u|_{\mathcal{E}, \mathcal{Q}_{\delta}, p}, & \left\|\mathcal{R}^{\prime} \wedge \mathbf{N}-\mathcal{R} \wedge \mathbf{N}\right\|_{L^{p}\left(\omega ; \mathbb{R}^{3}\right)} \leq C \delta^{-1 / p}|u|_{\mathcal{E}, \mathcal{Q}_{\delta}, p} \\ \left\|\frac{\partial \mathcal{U}^{\prime}}{\partial s_{\alpha}}-\frac{\partial \mathcal{U}}{\partial s_{\alpha}}\right\|_{L^{p}\left(\omega ; \mathbb{R}^{3}\right)} \leq C \delta^{-1 / p}|u|_{\mathcal{E}, \mathcal{Q}_{\delta}, p}, & \left\|\frac{\partial}{\partial s_{\alpha}}(\mathcal{R} \wedge \mathbf{N})-\frac{\partial}{\partial s_{\alpha}}(\mathcal{R} \wedge \mathbf{N})\right\| \leq C \delta^{-1 / p}|u|_{\mathcal{E}, \mathcal{Q}_{\delta}, p}\end{cases}
$$

The constants do not depend on $\delta$.

Proof . These estimates are immediate consequences of (3.4) and (3.14).

Let $\gamma_{0}$ be a part of $\partial \omega$ of measure non null and $\Gamma_{0 \delta}=\Phi\left(\gamma_{0} \times\right]-\delta, \delta[)$. If the displacement $u$ is null over $\Gamma_{0 \delta}$ then we have $\mathcal{U}=\mathcal{R}=0$ on $\gamma_{0}$. From (3.4) and Poincaré's inequality we deduce

$$
\left\|\mathcal{U}^{\prime}\right\|_{L^{p}\left(\gamma_{0} ; \mathbb{R}^{3}\right)}+\delta\left\|\mathcal{R}^{\prime}\right\|_{L^{p}\left(\gamma_{0} ; \mathbb{R}^{3}\right)} \leq C \delta^{1-2 / p}|u|_{\mathcal{E}, \mathcal{Q}_{\delta}, p}
$$

Corollary 2 . (Korn's inequality). We suppose that the shell is fixed over $\Gamma_{0 \delta}$. Then we have

$$
|u|_{\mathcal{D}, \mathcal{Q}_{\delta}, p}+\|\left. u\right|_{L^{p}\left(\mathcal{Q}_{\delta} ; \mathbb{R}^{3}\right)} \leq \frac{C}{\delta}|u|_{\mathcal{E}, \mathcal{Q}_{\delta}, p}
$$

The constant does not depend on $\delta$.

Proof . This is an immediate consequence of (3.14) and (3.18).

\section{Appendix}

We denote

- $\|x\|_{2}$ the euclidian norm of $x$,

- $\|\mid A\| \|_{2}$ the spectral norm of the matrix $A$,

- $B(a ; r)$ the ball centered at $a$ and of radius $r$,

- $I_{3}$ the unit matrix.

Lemma 4.1 : Let $\mathcal{U}$ be an open set included in $B(O ; R)$, star-shaped with respect to the ball $B\left(O ; R_{1}\right)$ and $\Phi$ the linear transformation

$$
\Phi(x)=A x+b \text { where } A \text { is an invertible matrix and where } b \in \mathbb{R}^{3} \text {. }
$$

The open set $\mathcal{V}=\Phi(\mathcal{U})$ is included in the ball $B\left(b ;\||| A\| \|_{2} R\right)$ and it is star-shaped with respect to the ball $B\left(b ; R_{1} /\left\|A^{-1}\right\|_{2}\right)$.

Proof . The open set $\mathcal{V}$ is star-shaped with respect to the points of the set $\Phi\left(B\left(O ; R_{1}\right)\right)$. This set is included in the ball $B\left(b ; R_{1} /\left\|A^{-1}\right\|_{2}\right)$.

Lemma 4.2 : Let $\mathcal{U}$ be an open set included in $B(O ; R)$, star-shaped with respect to the ball $B\left(O ; R_{1}\right)$ and $\Phi$ a $\mathcal{C}^{1}$ diffeomorphism from $\overline{\mathcal{U}}$ onto $\overline{\mathcal{V}}=\Phi(\overline{\mathcal{U}})$ verifying

$$
\Phi(O)=O \quad \text { and } \quad \forall x \in \overline{\mathcal{U}} \quad\left\|\nabla \Phi(x)-I_{3}\right\|_{2} \leq\left(\frac{R_{1}}{4 R}\right)^{3}
$$

The open set $\mathcal{V}=\Phi(\mathcal{U})$ is included in the ball $B(O ; 2 R)$ and is star-shaped with respect to the ball $B\left(O ; R_{1} / 4\right)$.

Proof . We put $c=\left(\frac{R_{1}}{4 R}\right)^{3}$. For any $(x, y) \in \overline{\mathcal{U}} \times \overline{\mathcal{U}}$ such that $[x, y] \in \overline{\mathcal{U}}$ we have

$$
\|\Phi(x)-\Phi(y)-(x-y)\|_{2} \leq c\|x-y\|_{2} \quad \Longrightarrow \quad\|\Phi(x)-x\|_{2} \leq c\|x\|_{2}
$$


The open set $\mathcal{V}$ is included in the ball $B(O ; 2 R)$ since $\|\Phi(x)\|_{2} \leq 2\|x\|_{2}$.

Now we prove that the open set $\mathcal{V}$ is star-shaped with respect to any point of the ball $B\left(O ; R_{1} / 4\right)$.

Let $A$ be a point in the ball $B\left(O ; R_{1} / 3\right)$. We prove by contradiction that the open segment joining $\Phi(A)$ to any point on the boundary of $\mathcal{V}$ is included in $\mathcal{V}$.

Let $y^{\prime}$ be a point on the boundary of $\mathcal{V}$. There exists $y \in \partial \mathcal{U}$ such that $y^{\prime}=\Phi(y)$. We put $A^{\prime}=\Phi(A)$. If the segment $\left[A^{\prime}, y^{\prime}\right.$ [ contains a point $z^{\prime}=\Phi(z)$ on the boundary of $\mathcal{V}$ then

$$
\left.z \in \partial \mathcal{U} \quad \text { and } \quad z^{\prime}=\lambda A^{\prime}+(1-\lambda) y^{\prime} \quad \lambda \in\right] 0,1[\text {. }
$$

From the second inequality in (4.1) we deduce that

$$
\left.\begin{array}{c}
\left\|A^{\prime}-A\right\|_{2}=\|\Phi(A)-A\|_{2} \leq c\|A\|_{2} \leq c R_{1} / 3 \\
\left\|y^{\prime}-y\right\|_{2}=\|\Phi(y)-y\|_{2} \leq c\|y\|_{2} \leq c R
\end{array}\right\} \Rightarrow\left\|z^{\prime}-(\lambda A+(1-\lambda) y)\right\|_{2} \leq c R
$$

Besides we get

$$
\|z-(\lambda A+(1-\lambda) y)\|_{2}=\left\|[z-\Phi(z)]+\left[z^{\prime}-(\lambda A+(1-\lambda) y)\right]\right\|_{2} \leq c\|z\|_{2}+c R \leq 2 c R \leq \frac{R_{1}}{32}
$$

Hence $z$ belongs to the coloured cylinder (see Figure 1). In Figure 2 we consider the plan containing $A, y$ and $z$. The point $z$ belongs to the exterior of the convex hull of the set $\overline{B\left(A ; R_{1} / 3\right)} \cup\{y\}$ (this convex hull minus $\{y\}$ is included in $\mathcal{U})$ and in the same way $y$ is outside the convex hull of the set $\overline{B\left(A ; R_{1} / 3\right)} \cup\{z\}$. Then we have $\|y-z\|_{2} \leq \frac{\|y-A\|_{2}}{10}$ and $\alpha \leq \widehat{z y A} \leq \pi-\alpha$.

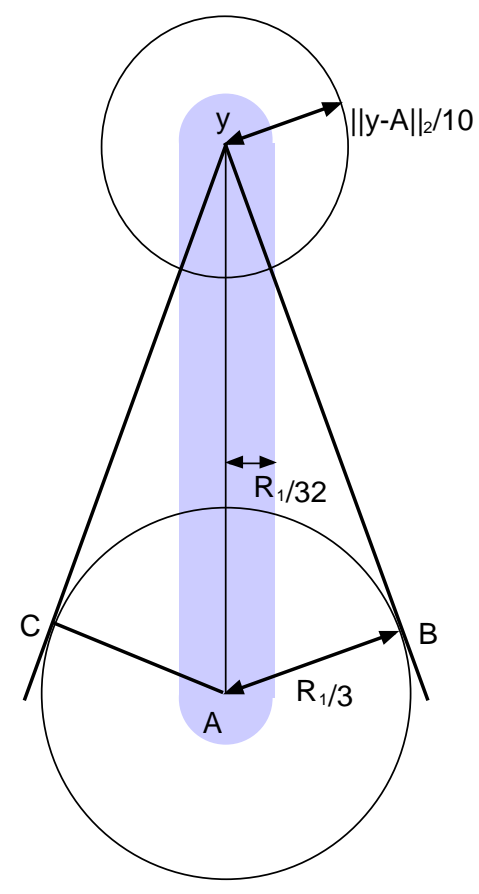

Figure 1.

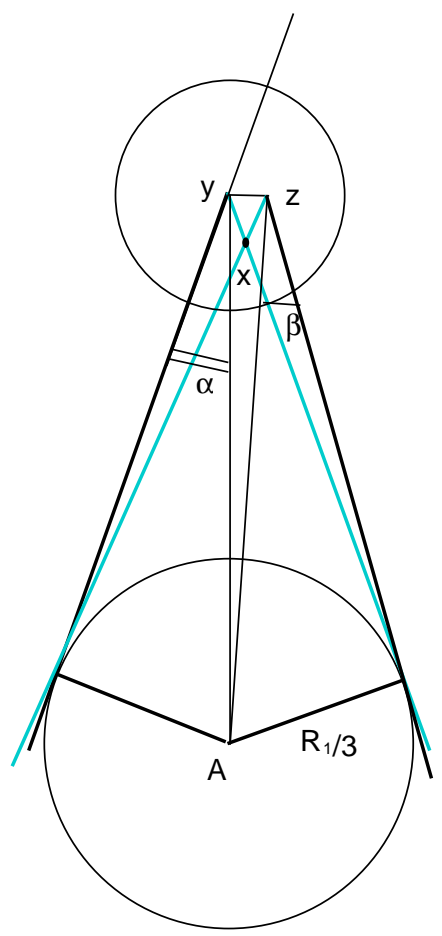

Figure 2. 
The open set $\mathcal{U}$ is star-shaped with respect to every point in the ball centered at $A$ and of radius $R_{1} / 3$. Moreover $\frac{2 R_{1}}{3} \leq\|y-A\|_{2} \leq R+\frac{R_{1}}{3} \leq \frac{4 R}{3}$ and $\|z-A\|_{2} \leq R+\frac{R_{1}}{3} \leq \frac{4 R}{3}$. Then we have

$$
\frac{R_{1}}{3} \frac{3}{4 R} \leq \sin (\alpha) \leq \frac{1}{2} \quad \text { and } \quad \frac{R_{1}}{3} \frac{3}{4 R} \leq \sin (\beta)
$$

Let $x$ be the common point to the two coloured lines. We have

$$
\|y-x\|_{2} \leq \frac{\|y-z\|_{2}}{\sin (2 \alpha)} \leq \frac{8 R}{3 R_{1}}\|y-z\|_{2} \quad \quad\|x-z\|_{2} \leq \frac{\|y-z\|_{2}}{\sin (2 \alpha)} \leq \frac{8 R}{3 R_{1}}\|y-z\|_{2}
$$

since $\sin (2 \alpha) \geq \frac{3 R_{1}}{8 R}$. The segments $[y, x]$ and $[x, z]$ are included in $\overline{\mathcal{U}}$ so, from the inequalities (4.1) we obtain

$$
\begin{aligned}
\|(\Phi(y)-\Phi(A)) \wedge & (\Phi(y)-\Phi(z)-(y-z))\left\|_{2} \leq\right\| \Phi(y)-\Phi(A)\left\|_{2}\right\| \Phi(y)-\Phi(z)-(y-z) \|_{2} \\
& \leq(1+c) \frac{4 R}{3}\|\Phi(y)-\Phi(z)-(y-z)\|_{2} \\
& \leq(1+c) \frac{4 R}{3}\|(\Phi(y)-\Phi(x)-(y-x))+(\Phi(x)-\Phi(z)-(x-z))\|_{2} \\
& \leq(1+c) \frac{4 R}{3} c\left\{\|y-x\|_{2}+\|x-z\|_{2}\right\} \leq c(1+c) \frac{64}{9} \frac{R^{2}}{R_{1}}\|y-z\|_{2}
\end{aligned}
$$

The points $y^{\prime}=\Phi(y), z^{\prime}=\Phi(z)$ and $A^{\prime}=\Phi(A)$ are on the same line, hence

$$
\begin{aligned}
\|\left(\Phi(y)-\Phi(A) \wedge(\Phi(y)-\Phi(z)-(y-z)) \|_{2}\right. & =\|(\Phi(y)-\Phi(A)) \wedge(y-z)\|_{2} \\
& =\|(y-A+[\Phi(y)-\Phi(A)-(y-A)]) \wedge(y-z)\|_{2} \\
& \geq\|(y-A) \wedge(y-z)\|_{2}-c \frac{4 R}{3}\|y-z\|_{2}
\end{aligned}
$$

We recall that $\|y-A\|_{2} \geq \frac{2 R_{1}}{3}$ and $\frac{R_{1}}{4 R} \leq \sin (\alpha) \leq \sin (\widehat{z y A})$. That gives

$$
\frac{R_{1}^{2}}{6 R}\|y-z\|_{2} \leq \frac{R_{1}}{4 R}\|y-z\|_{2}\|y-A\|_{2} \leq\|(y-A) \wedge(y-z)\|_{2} \leq c \frac{4 R}{3}\left[(1+c) \frac{16 R}{3 R_{1}}+1\right]\|y-z\|_{2} \quad \Longrightarrow \quad c \geq \frac{R_{1}^{3}}{60 R^{3}}
$$

which is a contradiction. The open set $\mathcal{V}$ is star-shaped with respect to the point $\Phi(A)$.

The ball $B\left(O ; R_{1} / 4\right)$ is included in the range by $\Phi$ of the ball $B\left(O ; R_{1} / 3\right)$ since for every $x^{\prime} \in \mathcal{V}$ we have

$$
\frac{1}{1+c}\left\|x^{\prime}\right\|_{2} \leq\left\|\Phi^{-1}\left(x^{\prime}\right)\right\|_{2} \leq \frac{1}{1-c}\left\|x^{\prime}\right\|_{2}
$$

Corollary of Lemmas 4.1 and 4.2 . Let $\mathcal{U}$ be an open set included in the ball $B(O ; R)$, star-shaped with respect to the ball $B\left(O ; R_{1}\right)$ and $\Phi$ a $\mathcal{C}^{1}$ diffeomorphism from $\overline{\mathcal{U}}$ onto $\overline{\mathcal{V}}=\Phi(\overline{\mathcal{U}})$ verifying

$$
\left.\forall x \in \overline{\mathcal{U}} \quad\left\|\left|\nabla \Phi(x)-\nabla \Phi(O)\left\|_{2} \leq\left(\frac{R_{1}}{4 R}\right)^{3}\right\|\right| \nabla \Phi(O)\right\|\left\|_{2}^{3}\right\|\left\|(\nabla \Phi(O))^{-1}\right\|\right|_{2} ^{2}
$$

The open set $\mathcal{V}=\Phi(\mathcal{U})$ is included in a ball of radius $2\|\mid \nabla \Phi(O)\| \|_{2} R$ and is star-shaped with respect to a ball of radius $R_{1} /\left(4 \mid\left\|(\nabla \Phi(O))^{-1}\right\|_{2}\right)$. 
Proof . We consider the mapping

$$
\Psi_{0}(x)=\Phi(O)+\nabla \Phi(O) x \quad x \in \overline{\mathcal{U}}
$$

The open set $\mathcal{W}=\Psi_{0}(\mathcal{U})$ is included in the ball $B\left(\Phi(O) ; R^{\prime}\right)\left(R^{\prime}=\|\| \nabla \Phi(O) \mid \|_{2} R\right)$ and it is star-shaped with respect to the ball $B\left(\Phi(O) ; R_{1}^{\prime}\right)\left(R_{1}^{\prime}=R_{1} /\left\|\mid(\nabla \Phi(O))^{-1}\right\|_{2}\right)$. Now let $\Psi_{1}$ be the mapping $\Psi_{1}=\Phi \circ \Psi_{0}^{-1}$. This map is a $\mathcal{C}^{1}$ diffeomorphism from $\overline{\mathcal{W}}$ onto $\overline{\mathcal{V}}$. We have $\Psi_{1}(\Phi(O))=\Phi(O)$ and $\Psi_{1}^{\prime}(\Phi(O))=I_{3}$. Moreover

$$
\begin{aligned}
\forall y \in \mathcal{W} \quad\left\|\mid \Psi_{1}^{\prime}(y)-I_{3}\right\|_{2} & \leq\|\| \nabla \Phi\left(\Psi_{1}^{-1}(y)\right)-\nabla \Phi(O)\|\|_{2}\left\|\mid(\nabla \Phi(O))^{-1}\right\|_{2} \\
& \leq\|\| \nabla \Phi\left(\Psi_{1}^{-1}(y)\right)-\nabla \Phi(O)\|\|_{2}\left\|\mid(\nabla \Phi(O))^{-1}\right\| \|_{2}=\left(\frac{R_{1}^{\prime}}{4 R^{\prime}}\right)^{3}
\end{aligned}
$$

The open set $\mathcal{V}$ is included in a ball of radius $R^{\prime}$ and it is star-shaped with respect to a ball of radius $R_{1}^{\prime} / 4$. $\square$

\section{Proof of Lemma 3.2 .}

We use again the mappings introduced in Step 1 of Theorem 3.1. Through the use of easy geometrical arguments we show that if $\delta \leq \inf \{C / 2, B / 4(M+1)\}$, we have

$$
\begin{aligned}
& \omega_{2 \delta} \backslash \omega \subset \bigcup_{r=1}^{K}\left\{\left(s_{1 r}, s_{2 r}\right) \mid f_{r}\left(s_{1 r}\right)<s_{2 r}<f_{r}\left(s_{1 r}\right)+2 \delta(M+1), \quad s_{1 r} \in\right] 0, A[\}, \\
& \{\widehat{s} \in \omega \mid \operatorname{dist}(\widehat{s}, \partial \omega)<2 \delta\} \subset \bigcup_{r=1}^{K}\left\{\left(s_{1 r}, s_{2 r}\right) \mid f_{r}\left(s_{1 r}\right)-2 \delta(M+1)<s_{2 r}<f_{r}\left(s_{1 r}\right), s_{1 r} \in\right] 0, A[\} .
\end{aligned}
$$

Now we extend the displacement $u \in W^{1, p}\left(\mathcal{Q}_{\delta} ; \mathbb{R}^{3}\right)$ near the portion of the boundary given by the mapping $f_{r}$. We begin with $r=1$. Without being detrimental to the general case we can suppose that $\left(O_{1} ; \mathbf{e}_{11}, \mathbf{e}_{21}\right)=\left(O ; \mathbf{e}_{1}, \mathbf{e}_{2}\right)$. In order to simplify the notation we replace $f_{1}$ by $f$.

We suppose also that $\delta \leq \inf \{A / 12, B /(18 M+2)\}$. Let $N$ be an odd integer belonging to $\left[\frac{2}{3} \frac{A}{2 \delta}, \frac{A}{2 \delta}\right]$. We set $\alpha_{k}=k \frac{A}{2 N}, k \in\{0, \ldots, 2 N\}$. We have $N$ greater than 3 and $\delta \leq \frac{A}{2 N} \leq \frac{3}{2} \delta$. For any $k \in\{0, \ldots, N-1\}$ the domains

$$
\begin{aligned}
& \left.\mathcal{B}_{\delta, k}=\left\{\left(s_{1}, s_{2}\right) \mid f\left(s_{1}\right)-2 \delta(9 M+1)<s_{2}<f\left(s_{1}\right), s_{1} \in\right] \alpha_{2 k}, \alpha_{2(k+1)}[\} \times\right]-\delta, \delta[ \\
& \left.\mathcal{B}_{\delta, k}^{\prime}=\left\{\left(s_{1}, s_{2}\right) \mid f\left(s_{1}\right)-2 \delta(9 M+1)<s_{2}<f\left(s_{1}\right)+2 \delta(9 M+1), s_{1} \in\right] \alpha_{2 k}, \alpha_{2(k+1)}[\} \times\right]-\delta, \delta[
\end{aligned}
$$

are star-shaped with respect to the ball of center $\left(\alpha_{2 k+1}, f\left(\alpha_{2 k+1}\right)+\delta(9 M+1), 0\right)$ and of radius $\delta / 2$ and they have a diameter less than $\delta(42 M+9)$. We put

$$
k \in\left\{0, \ldots, \frac{N-3}{2}\right\} \quad\left\{\begin{array}{l}
\mathcal{C}_{\delta, k}=\operatorname{interior}\left(\overline{\mathcal{B}_{\delta, 2 k}} \cup \overline{\mathcal{B}_{\delta, 2 k+1}} \cup \overline{\mathcal{B}_{\delta, 2 k+2}}\right) \\
\mathcal{C}_{\delta, k}^{\prime}=\operatorname{interior}\left(\overline{\mathcal{B}_{\delta, 2 k}^{\prime}} \cup \overline{\mathcal{B}_{\delta, 2 k+1}^{\prime}} \cup \overline{\mathcal{B}_{\delta, 2 k+2}^{\prime}}\right) \\
\mathcal{C}_{\delta, k}^{\prime \prime}=\operatorname{interior}\left(\overline{\mathcal{B}_{\delta, 2 k}^{\prime}} \cup \overline{\mathcal{B}_{\delta, 2 k+1}} \cup \overline{\mathcal{B}_{\delta, 2 k+2}^{\prime}}\right)
\end{array}\right.
$$

The open sets $\mathcal{C}_{\delta, k}$ and $\mathcal{C}_{\delta, k}^{\prime}$ are star-shaped with respect to the ball of center $\left(\alpha_{4 k+3}, f\left(\alpha_{4 k+3}\right)-\delta(9 M+1), 0\right)$ 
and of radius $\delta / 2$ and they have a diameter less than $(42 M+15) \delta=R \delta$.

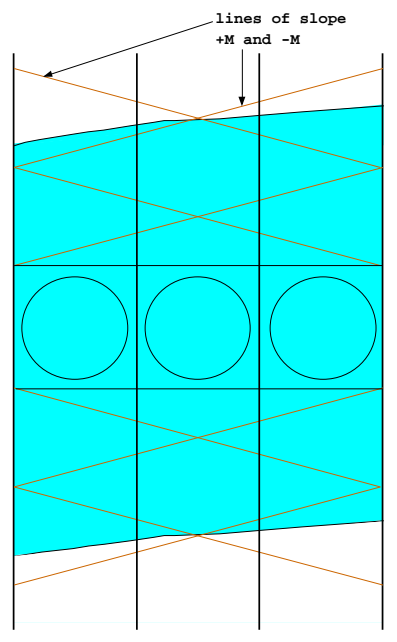

Figure 3. The domain $\mathcal{C}_{\delta, k} \cap\left\{s_{3}=0\right\}$

There exists $C_{0}>0$ which does not depend on $\delta$ such that

$$
\forall(s, \tilde{s}) \in \mathcal{C}_{\delta, k}^{\prime} \times \mathcal{C}_{\delta, k}^{\prime}, \quad\|\nabla \nabla \Phi(s)-\nabla \Phi(\tilde{s})\|_{2} \leq C_{0} \delta
$$

In the sequel we will work with the portions $\mathbb{B}_{\delta, k}=\Phi\left(\mathcal{B}_{\delta, k}\right), \mathbb{B}_{\delta, k}^{\prime}=\Phi\left(\mathcal{B}_{\delta, k}^{\prime}\right)$ and $\mathbb{C}_{\delta, k}^{\prime}=\Phi\left(\mathcal{C}_{\delta, k}^{\prime}\right)$ of the shell. Now we take $\delta$ such that

$$
\delta \leq \inf \left\{\frac{c_{0}^{3}}{C_{0} c_{1}^{2}}\left(\frac{1 / 2}{4 R}\right)^{3}, \delta_{0}\right\}
$$

Then we have

$$
C_{0} \delta \leq\left(\frac{1 / 2}{4 R}\right)^{3} \inf _{s \in \Omega_{\delta}}\left\{\|\nabla \nabla(s)\|\left\|_{2}^{3}\right\|(\nabla \Phi(s))^{-1} \mid \|_{2}^{2}\right\}
$$

Thanks to the corollary of Lemmas 4.1 and 4.2 the domains $\mathbb{B}_{\delta, k}$ have a diameter less than $3 R \delta$ and they are star-shaped with respect to a ball of radius $\delta / 16$. From Theorem 1.3 there exist rigid body displacements $r_{2 k}, k \in\left\{0, \ldots, \frac{N-1}{2}\right\}$ and $\tilde{r}_{k}, k \in\left\{0, \ldots, \frac{N-3}{2}\right\}$ such that

$$
\left\{\begin{array} { r l } 
{ \mathcal { D } _ { p } ( u - r _ { 2 k } , \mathbb { B } _ { \delta , 2 k } ) } & { \leq C \mathcal { E } _ { p } ( u , \mathbb { B } _ { \delta , 2 k } ) , } \\
{ \| u - r _ { 2 k } \| _ { L ^ { p } ( \mathbb { B } _ { \delta , 2 k } ; \mathbb { R } ^ { 3 } ) } ^ { p } } & { \leq C \delta ^ { p } \mathcal { E } _ { p } ( u , \mathbb { B } _ { \delta , 2 k } ) , }
\end{array} \quad \left\{\begin{array}{c}
\mathcal{D}_{p}\left(u-\tilde{r}_{k}, \mathbb{C}_{\delta, k}\right) \leq C \mathcal{E}_{p}\left(u, \mathbb{C}_{\delta, k}\right), \\
\left\|u-\tilde{r}_{k}\right\|_{L^{p}\left(\mathbb{C}_{\delta, k} ; \mathbb{R}^{3}\right)} \leq C \delta^{p} \mathcal{E}_{p}\left(u, \mathbb{C}_{\delta, k}\right),
\end{array}\right.\right.
$$

where the constants do not depend on $k$ and $\delta$.

We define the one-to-one mapping $\left.\Psi: \mathcal{C}_{\delta, k}^{\prime} \longrightarrow\right]-3,3[\times]-1,1[\times]-1,1[$ by

$$
\left(s_{1}, s_{2}, s_{3}\right) \longmapsto\left(\frac{s_{1}-\alpha_{4 k+3}}{\delta}, \frac{s_{2}-f\left(s_{1}\right)}{\delta(18 M+2)}, \frac{s_{3}}{\delta}\right)
$$

We have

$$
\forall s \in \mathcal{C}_{\delta, k}^{\prime} \quad \operatorname{det}(\nabla \Psi(s))=\frac{1}{(18 M+2) \delta^{3}}
$$


For every $k$ in $\left\{0, \ldots, \frac{N-1}{2}\right\}$, the function $\left(u-r_{2 k}\right) \circ \Phi$ is defined on $\mathcal{B}_{\delta, 2 k}$. We extend $\left(u-r_{2 k}\right) \circ \Phi \circ \Psi^{-1}$ by reflexion and then we return in $\mathbb{B}_{\delta, 2 k}^{\prime}$ and we add $r_{2 k}$ to the obtained displacement. We call again $u$ this new displacement. We have (the constants do not depend on $k$ and $\delta$ )

$$
\mathcal{E}_{p}\left(u, \mathbb{B}_{\delta, 2 k}^{\prime}\right)+\mathcal{D}_{p}\left(u-r_{2 k}, \mathbb{B}_{\delta, 2 k}^{\prime}\right) \leq C \mathcal{E}_{p}\left(u, \mathbb{B}_{\delta, 2 k}\right), \quad\left\|u-r_{2 k}\right\|_{L^{p}\left(\mathbb{B}_{\delta, 2 k}^{\prime} ; \mathbb{R}^{3}\right)}^{p} \leq C \delta^{p} \mathcal{E}_{p}\left(u, \mathbb{B}_{\delta, 2 k}\right)
$$
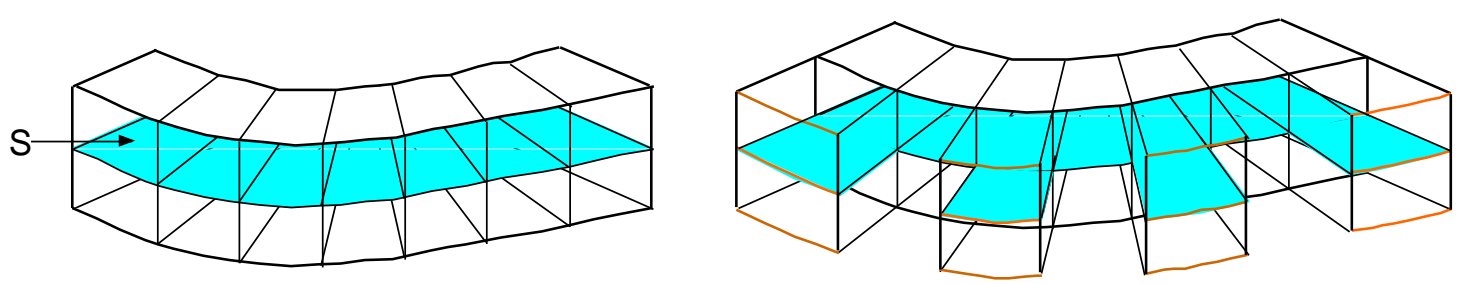

Figure 4. A portion of the boundary of the shell and the first extension of displacement $u$.

Thanks to (4.3) we can compare $\tilde{r}_{k}, r_{2 k}$ and $r_{2 k+2}$. We obtain

$$
\mathcal{E}_{p}\left(u, \mathbb{C}_{\delta, k}^{\prime \prime}\right)+\mathcal{D}_{p}\left(u-\tilde{r}_{k}, \mathbb{C}_{\delta, k}^{\prime \prime}\right) \leq C \mathcal{E}_{p}\left(u, \mathbb{C}_{\delta, k}\right), \quad\left\|u-\tilde{r}_{k}\right\|_{L^{p}\left(\mathbb{C}_{\delta, k}^{\prime \prime} ; \mathbb{R}^{3}\right)}^{p} \leq C \delta^{p} \mathcal{E}_{p}\left(u, \mathbb{C}_{\delta, k}\right)
$$

The function $\left(u-\tilde{r}_{k}\right) \circ \Phi$ is defined on $\mathcal{C}_{\delta, k}^{\prime \prime}$. There exists a linear and continuous extension operator $P$ from $W^{1, p}(]-3,3[\times]-1,1\left[{ }^{2} \backslash\right]-1,1[\times] 0,1[\times]-1,1[)$ into $W^{1, p}(]-3,3[\times]-1,1\left[^{2}\right)$. Using $P$ we extend $\left(u-\tilde{r}_{k}\right) \circ \Phi \circ \Psi^{-1}$ then we return in $\mathbb{C}_{\delta, 2 k}^{\prime}$ and we add $\tilde{r}_{k}$ to the obtained displacement. We call again $u$ this new displacement. We have

$$
\mathcal{E}_{p}\left(u, \bigcup_{k=0}^{(N-3) / 2} \mathbb{C}_{\delta, k}^{\prime}\right) \leq C \mathcal{E}_{p}\left(u, \bigcup_{k=0}^{(N-3) / 2} \mathbb{C}_{\delta, k}\right) \leq C \mathcal{E}_{p}\left(u, \mathcal{Q}_{\delta}\right)
$$

Now we go on with the other portions of the boundary of the shell. We take $r=2$. If the extension is already defined on a part of the domain $\left\{\left(s_{12}, s_{22}\right) \mid f_{2}\left(s_{12}\right)<s_{22}<f_{2}\left(s_{12}\right)+B, \quad s_{12} \in\right] 0, A[\}$, we preserve only the part defined on the first or on the last domain of type $\mathcal{B}_{\delta, k}^{\prime}$.

\section{References}

[1] M. Bernadou, P. G. Ciarlet and B. Miara. Existence theorems for two-dimensional linear shell theories, J. Elasticity 34 (1994), 111-138.

[2] D. Cioranescu, O.A. Oleinik and G. Tronel. Korn's inequalities for frame type structures and junctions with sharp estimates for the constants. Asymptotic Analysis, 8 (1994), 1-14

[3] P.G. Ciarlet and E. Sanchez-Palencia. An existence and uniqueness theorem for the two-dimensional linear membrane shell equations, J. Math. Pures Appl. 75 (1996), 51-67.

[4] P.G. Ciarlet. Mathematical Elasticity. Volume II : Theory of Plates. North-Holland, (1997).

[5] P.G. Ciarlet. Mathematical Elasticity. Volume III : Theory of Shells. North-Holland, (2000).

[6] $\mathrm{Ph}$. Destuynder. Une théorie asymptotique des plaques minces en élasticité linéaire. RMA 2, Masson (1986).

[7] G. Duvaut, J.L. Lions. Les Inéquations en Mécanique et en Physique. Dunod, Paris (1972).

[8] D. Gilbarg and N. Trudinger. Elliptic Partial Differential Equations of Second Order. Springer-Verlag (2001).

[9] G. Friesecke, R. D. James and S. Müller. A theorem on geometric rigidity and the derivation of nonlinear plate theory from the three-dimensional elasticity. Communications on Pure and Applied Mathematics, Vol. LX, 1461-1506 (2002). 
[10] G. Griso. Asymptotic behavior of curved rods by the unfolding method, Math. Meth. Appl. Sci. (2004); $27: 2081-2110$.

[11] G. Griso. Asymptotic behavior of structures made of plates. C. R. Acad. Sci. Paris, Ser. I 336 (2003), 101-106.

[12] G. Griso. Comportement asymptotique d'une grue. C. R. Acad. Sci. Paris, Ser. I 338 (2004), 261-266.

[13] G. Griso. Décomposition des déplacements d'une poutre. Simplification d'un problème d'élasticité. C. R. Acad. Sci. Paris, Sér. II 333 (2005), 475-480.

[14] G. Griso. Asymptotic behavior of structures made of plates. Analysis and Applications, Vol. 3, Nr 4 (2005), 325-356

[15] G. Griso. Obtention d'équations de plaques par la méthode d'éclatement appliquéee aux équations tridimensionnelles. C. R. Acad. Sci. Paris, Ser. I, 343 (2006), 361-366

[16] G. Griso. Asymptotic behavior of structures made of curved rods. (To appear)

[17] M. Jurak and J. Tambača. Derivation and justification of a curved rod model. Math. Models Methods Appl. Sci. 9, 7 (1999), 991-1014.

[18] A. Kondratiev and O.A. Oleinik. Boundary value problems for the theory of elasticity system in unbounded domains. Korn's inequalities, Uspekhi Mat. Nauk., 43, 5 (1988), 55-98.

[19] A. Kondratiev and O.A. Oleinik. On Korn's inequalities. C. R. Acad. Sci. Paris, Ser. I 308 (1989), 483-487.

[20] J. Nečas. Les Méthodes directes en Théorie des Equations Elliptiques, Masson (1967).

[21] J. A. Nitsche. On Korn's second inequality, RAIRO Analyse Numérique 15 (1981) 237-248.

[22] J. Sanchez-Hubert and E. Sanchez-Palencia. Introduction aux méthodes asymptotiques et à l'homogénéisation, Masson (1992).

[23] J. Sanchez-Hubert and E. Sanchez-Palencia. Statics of curved rods on account of torsion and flexion. European Jour. Mechanics A/Solids, 18 (1999), 365-390.

[24] L. Trabucho and J. M. Viano. Mathematical modeling of rods, in Handbook of Numerical Analysis. 487-974, ed. P.G. Ciarlet and J.L. Lions, North-Holland, Amsterdam, 1996.

Georges GRISO

Laboratoire Jacques-Louis Lions

CNRS \& Université Pierre et Marie Curie (Paris VI)

Boîte postale 187

4, Place Jussieu

75252 Paris Cedex $05 \quad$ France

Email : georges.griso@wanadoo.fr 